\title{
Is infant-directed speech interesting because it is surprising? - Linking properties of IDS to statistical learning and attention at the prosodic level
}

\author{
Okko Räsänen ${ }^{1, *}$, Sofoklis Kakouros ${ }^{1} \&$ Melanie Soderstrom ${ }^{2}$ \\ ${ }^{1}$ Dept. Signal Processing and Acoustics, Aalto University, P.O. Box 12200, 00076, \\ AALTO, Finland \\ ${ }^{2}$ Department of Psychology, University of Manitoba, P404 Duff Roblin Building, \\ Winnipeg, MB R3T 2N2, Canada
}

*The corresponding author. Email: okko.rasanen@aalto.fi, GSM: +358-50-5911879. Other authors' emails: sofoklis.kakouros@aalto.fi, M_Soderstrom@umanitoba.ca 


\begin{abstract}
The exaggerated intonation and special rhythmic properties of infant-directed speech (IDS) have been hypothesized to attract infants' attention to the speech stream. However, there has been little work actually connecting the properties of IDS to models of attentional processing or perceptual learning. A number of such attention models suggest that surprising or novel perceptual inputs attract attention, where novelty can be operationalized as the statistical (un)predictability of the stimulus in the given context. Since prosodic patterns such as F0 contours are accessible to young infants who are also known to be adept statistical learners, the present paper investigates a hypothesis that F0 contours in IDS are less predictable than those in adult-directed speech (ADS), given previous exposure to both speaking styles, thereby potentially tapping into basic attentional mechanisms of the listeners in a similar manner that relative probabilities of other linguistic patterns are known to modulate attentional processing in infants and adults. Computational modeling analyses with naturalistic IDS and ADS speech from matched speakers and contexts show that IDS intonation has lower overall temporal predictability even when the F0 contours of both speaking styles are normalized to have equal means and variances. A closer analysis reveals that there is a tendency of IDS intonation to be less predictable at the end of short utterances, whereas ADS exhibits more stable average predictability patterns across the full extent of the utterances. The difference between IDS and ADS persists even when the proportion of IDS and ADS exposure is varied substantially, simulating different relative amounts of IDS heard in different family and cultural environments. Exposure to IDS is also found to be more efficient for predicting ADS intonation contours in new utterances than exposure to the equal amount of ADS speech. This indicates that the more variable prosodic contours of IDS also generalize to ADS, and may therefore enhance prosodic learning in infancy. Overall, the study suggests that one reason behind infant preference for IDS could be its higher information value at the prosodic level, as measured by the amount of surprisal in the F0 contours. This provides the first formal link between the properties of IDS and the models of attentional processing and statistical learning in the brain. However, this finding does not rule out the possibility that other differences between the IDS and ADS also play a role.
\end{abstract}

Keywords: language acquisition; infant-directed speech; statistical learning; attention; stimulus predictability; prosody 


\section{Introduction}

Infant-directed speech (IDS) is a speaking style that talkers often use when interacting with young infants. In contrast to adult-directed speech (ADS), IDS tends to have exaggerated intonational contours with higher fundamental frequency (F0) and larger frequency range (e.g., Grieser \& Kuhl, 1988), hyperarticulated vowels (Kuhl et al., 1997; but see also Martin et al., 2015), and shorter utterances with a higher token/type ratio (Phillips, 1973). Particular timbral characteristics have also been recently identified (Piazza, Iordan \& Lew-Williams, 2017). In addition to serving as language input tuned to the developmental stage of the listener (e.g., Snow, 1977), one hypothesized role of the exaggerated nature of IDS is that it may engage infants' attention to the speech stream more efficiently than ADS (e.g., Garnica, 1977; Fernald, Taeschner, Dunn, Papousek, de Boysson-Bardies \& Fukui, 1989; Thiessen, Hill \& Saffran, 2005, see also Soderstrom, 2007, for an overview), thereby facilitating language learning from speech. In support of this, a systematically higher attentional preference and pre-linguistic and linguistic interactional engagement towards IDS speakers was found in a recent meta-analysis conducted by Spinelli, Fasolo and Mesman (2017).

Although the exaggerated intonation of IDS is often implicitly assumed to be the cause for higher attentional attractiveness, according to our knowledge, no study has systematically evaluated properties of IDS in the context of what is known about perceptual mechanisms for stimulus-driven attention. Instead, the evidence for higher attentional capture of IDS largely comes from behavioral studies that show that infants prefer to listen to IDS over ADS (Fernald, 1985; Cooper \& Aslin, 1990; Pegg, Werker $\&$ McLeod, 1992). In addition, based on acoustic analyses and their perceptual correlates, IDS is often characterized as more salient or prominent than ADS, therefore also potentially being more interesting to the listeners (e.g., Garnica, 1977; Fernald et al., 1989; Soderstrom, 2007). However, the existing work has not explicated the concept of saliency or the nature of the link between saliency and attention in a manner that would enable concrete predictions of what counts as attention-capturing speech and why. In addition, it appears that larger magnitudes or variations in standard acoustic correlates of prosody do not necessarily map to higher attentional capture of the speech. For instance, Singh, Morgan, and Best (2002) found that IDS with higher and more variable pitch was not enough to capture infants' attention over emotional speech.

Since stimulus-driven attention and prominence of sensory input seem both to be connected to the unpredictability of the stimuli in the given context (see the next sub-section; see also Kidd, Piantadosi \& Aslin, 2012, 2014), it would be parsimonious to assume that the greater attention-capturing 
properties of IDS over ADS would be also based on probabilistic expectations. In other words, existing knowledge suggests that one reason why IDS might be more attractive to the listeners is simply because it exhibits different predictability properties than ADS at the level of prosodic features, given earlier exposure to speech in both speaking styles. For instance, larger variability of F0 in IDS already implies, but does not guarantee ${ }^{l}$, higher uncertainty regarding the realization of the intonation at any moment in time. However, no study has systematically compared the prosodic predictability of IDS and ADS from a statistical learning point of view. This is despite the fact that infants are known to be sensitive to statistical regularities in their perceptual experience (c.f., Saffran et al., 1996; Saffran, 2003; Soderstrom et al., 2009, and references therein) and to the prosodic structure of their native language already from an early age (e.g., Nazzi et al., 1998).

In the present paper, a quantitative investigation is carried out in order to test whether IDS intonation is indeed not just more variable, but also less predictable than ADS, thereby being in line with the predictability-based accounts of perceptual attention. Importantly, we assume that the listener is able to learn the typical behavior of intonational contours from speech experience, and this creates the basis for prosodic expectations for new speech input. In order to do this, a straightforward computational model of statistical learning is applied to F0 trajectories of naturalistic IDS and ADS and tested in its ability to predict intonational contours on speech utterances from both speaking styles.

\subsection{Stimulus-driven attention and statistical learning}

A number of models for stimulus-driven perceptual attention suggest that attention is drawn to stimuli that are low-probability, or unpredictable, in the given context (Itti \& Baldi, 2009; Zhang et al., 2008; Tsuchida \& Cottrell, 2012; Zarcone et al., 2016). From this viewpoint, attentional processing can be seen as a mechanism that enables the perceptual system to focus on aspects of the environment with high information content (Shannon, 1948), that is, input that is not yet learned and thereby accurately predicted by the brain (cf., Helmholtz, 1860; Ranganath \& Rainer, 2003; Friston, 2010; Clark, 2013). In addition, all behavioral paradigms for testing infant learning in experimental settings are based to a greater or lesser extent on some kind of preferential sampling of the environment based on the current knowledge of the infant. This inherently couples familiarity with external stimuli to the resulting attentional behavior. However, infants are also known to prefer visual and auditory stimuli that are surprising or novel only as long as the input is not too unlikely in the given context. This phenomenon is

\footnotetext{
${ }^{1}$ Unless speech is assumed to be a normally distributed IID process without temporal contiguity, a larger F0 range does not guarantee lower temporal predictability (cf., e.g., a simple sine wave, which is equally predictable regardless of amplitude or frequency).
} 
also known as the Goldilocks effect (Kidd et al., 2012, 2014) where the preferred degree of predictability is not too much or too little. This suggests that instead of just preferring the most unlikely inputs in each situation, the input stream should still be structured enough to support learning-a prerequisite for experience-based evaluation of the relative information value of different competing inputs.

Predictability-based accounts also have a notable role in several aspects of human language processing (e.g., Jurafsky, 1996; Jurafsky, Bell, Gregory \& Raymond, 2001; Watson, Arnold \& Tanenhaus, 2008). In the context of prosody, earlier work has demonstrated the role of prosodic prominence in the regulation of information rate in speech (e.g., Aylett \& Turk, 2004) and, e.g., tight coupling of lexical and syntactic analysis and prosodic parsing of the speech stream (e.g., Steinhauer, Alter \& Friederici, 1999; Buxó-Lugo \& Watson, 2016). In addition, recent work in adult speech perception suggests that low-probability intonation patterns in the context of otherwise predictable prosody are associated with higher perceptual prominence of the concurrent words (Kakouros \& Räsänen, 2016). Moreover, low-probability prosodic events seem to alter the semantic processing of the speech (e.g., Magne et al., 2005; Kakouros, Salminen \& Räsänen, 2018) similarly to low-probability lexical items in a predictive context (e.g., Cole, Mo \& Hasegawa-Johnson, 2010). Recent preliminary findings also suggest that adult listeners are sensitive, and rapidly adapt, to changing statistical properties of the intonation patterns and this leads to experience-based expectations for prosody (Kakouros et al., 2018). When these expectations are violated, the subjective impression of prominence gets enhanced and the semantic processing of the stimulus becomes altered (Kakouros et al., 2018). Overall, the earlier research indicates that auditory attention and perceptual prominence are connected to the predictability of the prosodic patterns, and this may also play a role in the perception of IDS.

Importantly, the concept of predictability necessitates some type of mechanism for learning regularities from experience, thus connecting attention and prominence with the concept of statistical learning. The most parsimonious assumption would be that prosodic learning utilizes the same statistical learning mechanisms hypothesized to play a role in other aspects of language acquisition, but now operating at the level of prosodic features such as F0 contours and energy envelopes instead of the phonemic units of the language. Since infants are known to be adept statistical learners of various linguistic and non-linguistic regularities, since it has long been known that prosodic cues are perceptually accessible to them (e.g., Kuhl \& Miller, 1982; Hirsh-Pasek, Kemler Nelson, Jusczyk, Cassidy, Druss \& Kennedy, 1987), and since they also seem to be sensitive to distributional properties 
of non-native tonal patterns at least at the age of 11-12 months (Liu \& Kager, 2014), it is reasonable to assume that infants are also sensitive to the general statistical structure of speech prosody.

If predictability of the stimulus is a major factor in controlling stimulus-driven attention in infants, as exemplified by the widely used preferential head-turn or looking-time paradigms to probe infants' learning, and if predictability also plays a role in infants' behavioral preferences for IDS, we would expect IDS to have different predictability properties than ADS. In the present study, we will look into one specific aspect of IDS, namely, intonation, and test how well F0 contours can be predicted over time for the two speaking styles in question.

\section{Data}

The speech material used in the present experiments comes from the ManyBabies study that aims to replicate IDS preference across a large number of labs (The ManyBabies Consortium, 2017). In the context of that study, naturalistic speech from female caregivers to their infants or from caregivers to other adults was recorded in central Canada and Northeastern US. All caregivers had infants aged 122250 days. The recordings were carried out in an infant-friendly greeting area/testing room using lapel clip-on microphones connected to smartphones. The task involved describing a closed set of labeled objects by asking the mother to take each object out of a bag one at a time and talk about it to her baby (IDS) or to an experimenter (ADS). In addition, there were two types of objects: those assumed to be familiar to the infants (e.g., a ball or a block) and those considered as novel (e.g., a sieve or a whisk). After rough manual segmentation of the recordings into utterances, the utterances were also classified into three categories: those containing the familiar object word, those containing the unfamiliar object word, and utterances without naming of the object.

In the present study, we used the Canadian section of the recordings, containing speech from a total of 11 mothers. The US recordings (4 mothers) were excluded due to significant presence of room reverberations and otherwise differing recording conditions that could have impacted our acoustic analyses. Utterances from all three categories (familiar, unfamiliar, no label) were included in the data. Four utterances with less than three segments with valid F0 estimates (see Methods) were discarded, leading to a total of $N=1070$ utterances (673 IDS, 397 ADS) with an average of $97.3 \pm 35.6$ utterances per talker. In the resulting data, the average utterance length was $1.7 \pm 1.1 \mathrm{~s}$ for IDS and $4.1 \pm 3.1 \mathrm{~s}$ for ADS when measured from the beginning of the first syllable to the end of the last syllable (see below). 


\section{Methods}

The overall goal of the analysis was to compare predictability of F0 trajectories in the IDS and ADS utterances using a statistical model. The model was first trained on intonation contours from both speaking styles, thereby simulating a child's exposure to different styles of speaking (e.g., overheard speech), and then tested in its capability to predict intonation in novel utterances spoken either in IDS or ADS style. The modeling was done by first estimating F0 trajectories for all speech, segmenting the speech into short-term temporal segments, parametrizing F0 trajectories during each segment, clustering the syllable-specific parameters into a finite number of discrete categories ("F0 shapes") in an unsupervised manner, and then modeling the temporal evolution of these F0 shapes across time. By training a predictive model from a set of utterances and then computing the likelihoods of F0 trajectories on a set of held-out utterances, statistical descriptors of F0 predictability could be estimated from the data. These descriptors, such as average utterance-level F0 predictability, were then compared between IDS and ADS from the same talkers during the object description task (Section 2). Fig. 1 shows a block diagram of the processing pipeline for an individual utterance. All experiments and analyses were conducted in MATLAB unless mentioned otherwise.

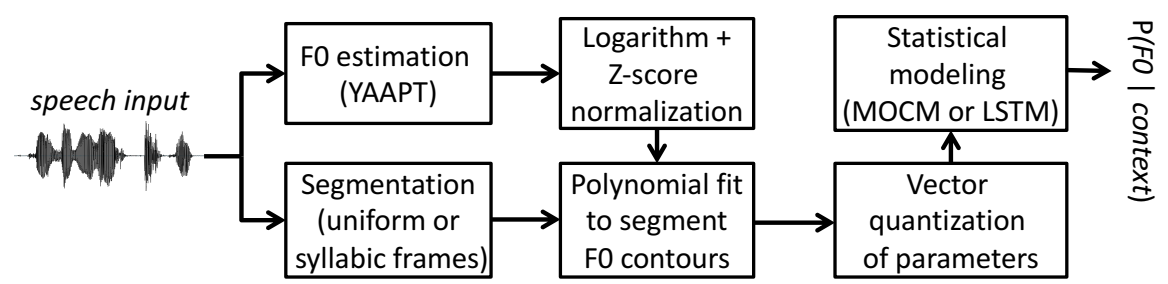

Figure 1: A schematic view of the F0 predictability analysis. The output is the probability of F0 in segment $s$ given the observed F0 in $m$ preceding segments (after training the statistical model on a number of other utterances).

\subsection{Pre-processing of F0 trajectories}

F0 trajectories were estimated at a 100-Hz sampling rate with YAAPT-algorithm (Zahorian \& Hu, 2008; version 4.0), constraining F0 estimates to the range of 120-600 Hz. YAAPT's ptch_fix() tool was used to post-process the pitch tracks for potential estimation errors and to perform shape-preserving piecewise cubic interpolation of the trajectories across unvoiced regions. 30-ms median filtering was also applied to the resulting pitch tracks to remove single outlier values. For the predictability analysis, utterance-level F0 tracks were transformed into logarithmic scale and then z-score normalized to zero mean and unit variance in order to focus on scale-free temporal behavior of the pitch trajectories (see 
left panel in Fig. 3 for the resulting distributions for IDS and ADS). In addition, the original nonnormalized and non-interpolated F0 contours were used as baseline features in the analyses.

As for the temporal segmentation, we compared two approaches: 1) syllable-based segments where F0 modeling was constrained by the rhythmic properties of speech, and 2) uniformly spaced segments where F0 was sampled and predicted at regular intervals. The former can be considered to be justifiable based on two perspectives: that of speech production where prosodic phenomena such as F0 contours tend to be tied to the underlying syllabic frames, and also speech perception where syllabic units or the closely related envelope-driven cortical oscillations are believed to play a central role in infant (e.g., Jusczyk, 1993; Mehler, Dupoux \& Segui, 1990; Swingley, 2005) and adult (e.g., Ghitza, 2011; Giraud \& Poeppel, 2012; Gross et al., 2013) processing of incoming speech. On the other hand, fixed-frame analysis may provide more consistent data for predictive modeling due to the absence of any potential syllabification errors (see below) and by removing any temporal variation in the predictive context or distance up to which the F0 predictions are made. In addition, fixed-frame windowing decouples the temporal sampling from the underlying linguistic content in the signals, which is not the case for syllable-timed sampling. This is particularly important, given the reported differential effects of utterance-final lengthening on IDS and ADS (e.g. Albin \& Echols, 1996; Bernstein Ratner, 1986; Church et al., 2005).

All utterances were syllabified automatically using a sonority envelope-based syllable segmentation model described in Räsänen, Doyle, and Frank (2018). All syllables preceding the utterance onsets or following utterance offsets, as defined by reliable F0 estimates from YAAPT, were discarded, leading to a total of 13903 syllables in the data set. Note that although this type of acoustic syllabification is not perfectly accurate in terms of the phonological rules of the language, it still provides systematic and speaking style agnostic chunking of speech into syllable-like units with each unit consisting of a sonorous peak surrounded by less-sonorous onsets and coda (see also, e.g., Villing, Ward \& Timoney, 2006, and references therein). Importantly, such acoustics-based chunking can be argued to better match the syllabification capabilities of pre-linguistic infants, since infants also have to rely on non-phonological acoustic cues in their perception of speech before they master the sound system of their native language (Räsänen et al., 2018). After obtaining the final syllable boundaries, every syllable was divided into two uniform-length sub-segments to provide a more detailed picture of the F0 dynamics within each syllable. As for the fixed-frame segmentation, 100-ms non-overlapping segments were used in order to maintain a similar temporal resolution and boundary conditions as with 
the syllable-based segments. Also in this case, voicing information was used to determine the first and last valid segment of each utterance.
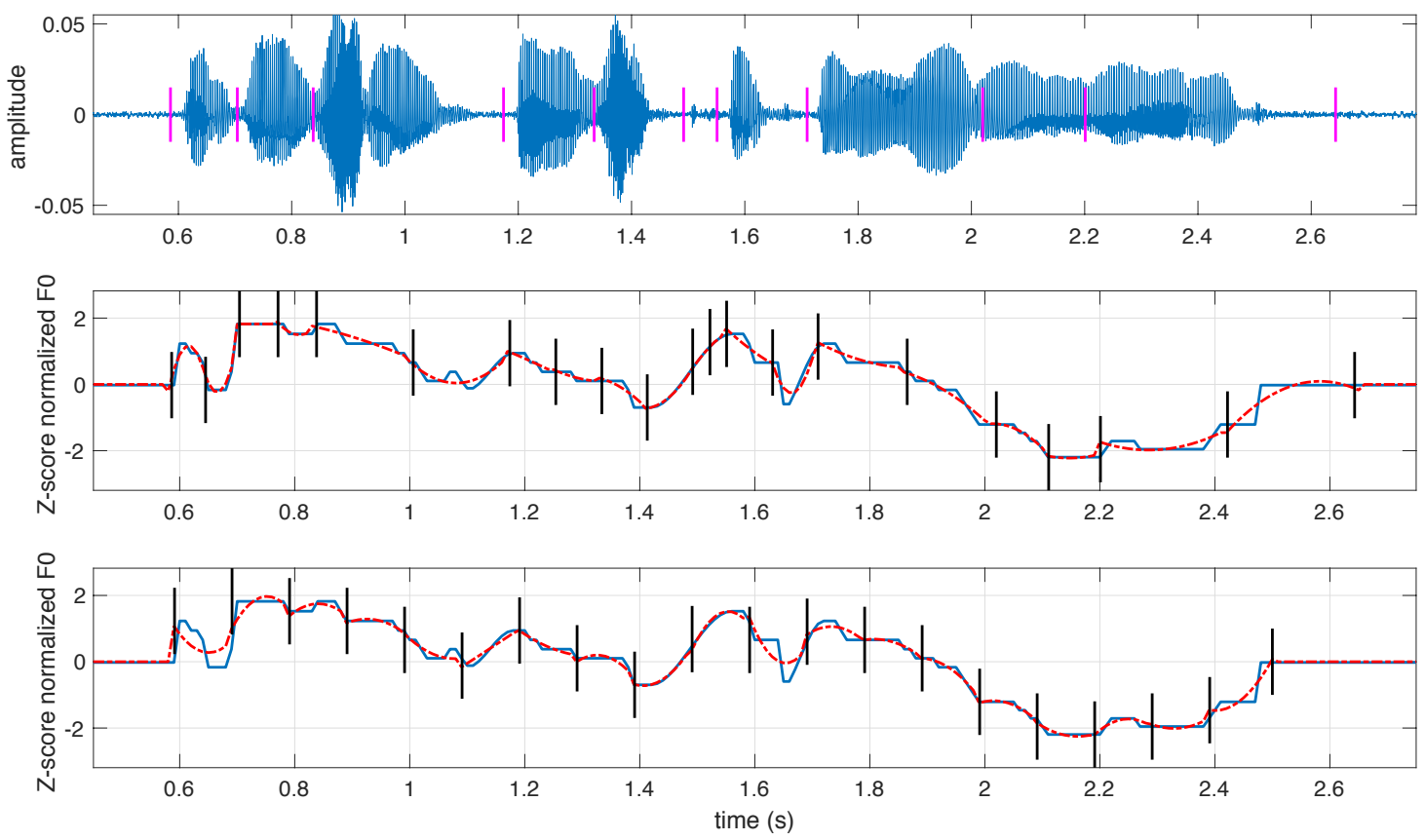

Figure 2: An example of segment-wise $2^{\text {nd }}$ order polynomial approximation of the F0 trajectory. Top: The original speech waveform with automatically estimated syllable boundaries with vertical lines. Middle: YAAPTestimated and z-score normalized $\log (\mathrm{F} 0)$ trajectory with interpolation across unvoiced signal regions (blue solid line) and the corresponding $2^{\text {nd }}$ order polynomial least-squares fit for F0 during each $1 / 2$ syllable (red dashed line). Segment boundaries are shown with vertical lines. Bottom: Same as the middle panel but now with 100-ms fixedlength segments.

Following the segmentation, F0 trajectories during each segment were parametrized by fitting a second order polynomial to the trajectory in time (Fig. 2). The polynomial coefficients without the constant term were then used as a parametric description of the F0 during the segment (see Fig. 3 right panel for coefficient distributions for syllabic frames). Parameters across all segments in the data were then vector quantized into $Q$ discrete categories. This was done by first applying standard k-means clustering with random initialization to the training data, and then finding the nearest cluster $q \in Q$ for each sample in the training and test sets (see below for data division). In practice, these $Q$ shapes correspond to different F0 patterns with varying curvature and rate of change as a function of time, a larger $Q$ simply meaning more fine-grained distinction between F0 patterns that occur during the segments. 

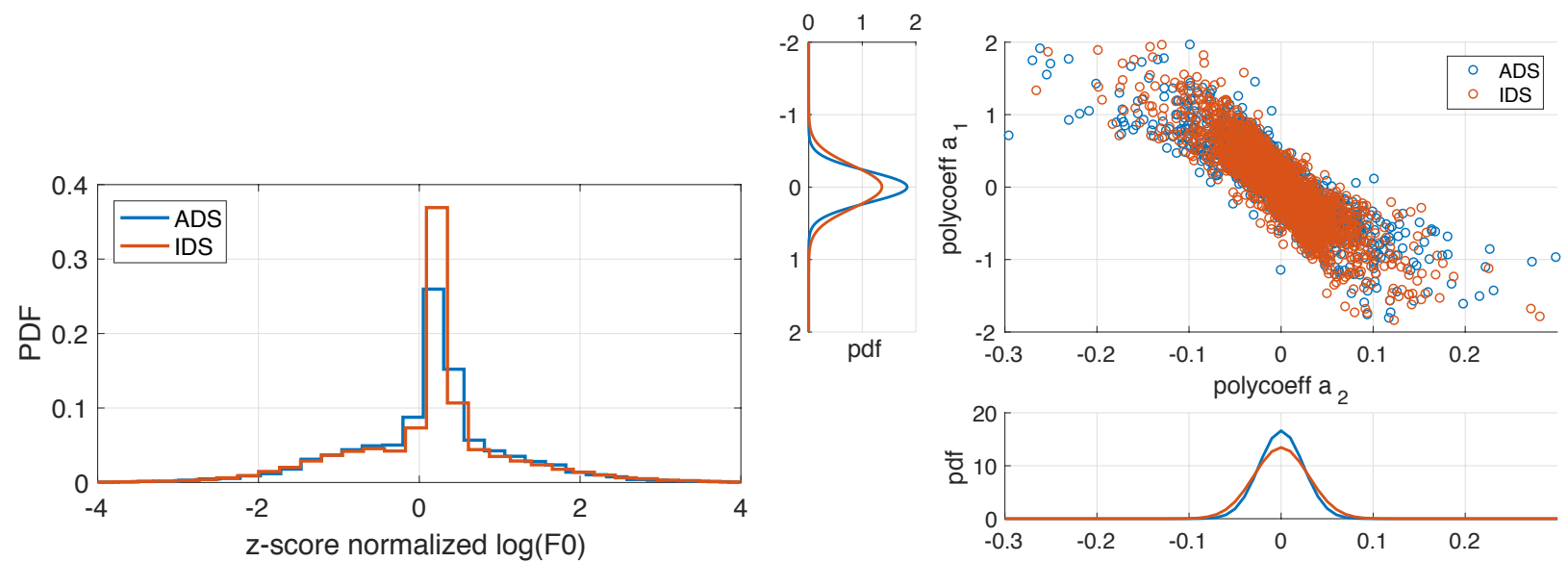

Figure 3: Left: Histogram distributions of z-score normalized $\log (\mathrm{F} 0)$ values for IDS and ADS across the entire dataset. After normalization, both speaking styles have highly similar distributions for context-independent measurements. Note that a substantial proportion of values are close to 0 due to the interpolation of the F0 trajectories across unvoiced regions. Right: Scatter plot of second-order polynomial coefficients without intercept, fit to normalized F0 contours (syllabic temporal segments), and used as input to the quantization of F0 trajectories. Marginal distributions (normal distributions fit to the data) are also shown for easier comparison. IDS exhibits a slightly higher variance of F0 shapes during each segment (slope $a_{1}$ and curvature $a_{2}$ ), but the overall distributions are highly similar for the two speaking styles.

\subsection{Temporal modeling of F0 state sequences}

As a result of the pre-processing, the F0 trajectory of each utterance was described as a sequence of discrete states $q_{s} \in Q$, one state per segment $s$. In order to quantify the predictability of F0, we investigated two alternative models to capture temporal regularities in the pitch tracks: mixed-order Markov chain model (MOCM; Saul \& Pereira, 1997) and Long Short-Term Memory neural networks (LSTM; Hochreiter \& Schmidhuber, 1997). Instead of computing $n$-gram statistics for different $n$-gram orders and then choosing and/or merging the models with the best predictive capability (where 2-grams would correspond to standard transitional probabilities), MOCM and LSTM both provide a principled way to model varying-order Markov processes with a single set of model parameters. Since they can also capture long-term dependencies in the data, they are especially suitable for modeling the present type of F0 sequences where both short- and long-term dependencies may exist in parallel.

In MOCM, the probability of an F0 shape $q_{s}$ in segment $s$, given the preceding $m$ segments, is calculated as 


$$
\begin{aligned}
& P\left(q_{s} \mid q_{s-1}, \ldots, q_{s-m}\right)= \\
& \sum_{k=1}^{m} \lambda_{k}\left(q_{s-k}\right) \mathbf{M}_{k}\left(q_{s-k}, q_{s}\right) \prod_{j=1}^{k-1}\left[1-\lambda_{j}\left(q_{s-j}\right)\right]
\end{aligned}
$$

where lag-specific transition matrices $\mathbf{M}$ and transition weights $\boldsymbol{\lambda}$ are estimated from training data using the Expectation Maximization (EM) algorithm (Saul \& Pereira, 1997). In the context of the present study, $\mathbf{M}_{k}$ describes the transition probabilities between segmental F0 contours at different lags $k$ while $\lambda$ weighs these probabilities from different distances based on the reliability of the probability estimates in the context of the observed shapes. In the experiments, MOCM model order was always set to $m=5$. We used our own MATLAB implementation² of MOCM, originally used in Räsänen \& Saarinen (2016).

LSTM is essentially a recurrent neural network (RNN) where hidden layer or layers of the network can capture temporal dependencies in the data by storing information on the preceding samples into the hidden activations of the network. In contrast to standard RNNs, LSTMs do not suffer from the problem of vanishing gradients during error backpropagation (training of the network). This enables them to learn statistical dependencies across long temporal distances, and therefore they are widely utilized in the current state-of-the-art machine learning systems for modeling and predicting temporal data. In the present experiments, the input to the LSTM was always 10 previous F0 shapes (segments) $q_{s-10 \ldots} q_{s-1}$ and the output was a posterior distribution $P\left(q_{s} \mid q_{s-1}, \ldots q_{s-10}\right)$ over the next element in the sequence, i.e., corresponding to left hand side of Eq. (1) with $m=10$. The actual implementation consisted of an embedding layer that transformed integer sequences into distributed continuous 30dimensional vectors, a single LSTM hidden layer with 30 units using sigmoid activation function, and an output layer with softmax activation that maps the output from the LSTM-layer into a probability distribution proper. The LSTM model was implemented using Keras/Theano libraries for deep learning (Chollet, 2015), trained using RMSprop as the optimizer, categorical cross-entropy as the loss criterion, using mini-batch size of 10 , and running the training until no improvement in model fit to the training data was observed.

In the experiments, MOCM and LSTM models were trained using the F0 sequences from a combined pool of IDS and ADS utterances and using style-balanced training. Balancing was done by first sampling a held-out test set consisting of $10 \%$ of all IDS and ADS utterances. Then the remaining $90 \%$ of the IDS utterances were used for the training together with a randomly sampled subset of the remaining ADS utterances so that the overall duration of the IDS and ADS training data was ensured to

\footnotetext{
${ }^{2}$ MATLAB scripts used in our experiments, including the MOCM implementation, are available for download at http://github.com/orasanen/IDS_predictability
} 
be equal $^{3}$. The reason for choosing an equal amount of IDS and ADS for the main analyses was that we wanted to ensure that any differences we might observe in the results are not simply driven by the amount of training data for the speaking style in question. In contrast, we first wanted to focus on the acoustic and temporal characteristics of the two speaking styles without any additional confounding factors. Since the ManyBabies corpus is not very large, it is likely that the presently used relatively powerful statistical models would benefit from additional training data beyond what is available in the corpus, resulting in a higher prediction accuracy for the style for which more data are available. In a follow-up experiment reported in Section 5.2, we relax this constraint and explore the impact of different proportions of IDS and ADS in the training to study whether the main findings also generalize to the typical proportions of IDS heard in different language environments.
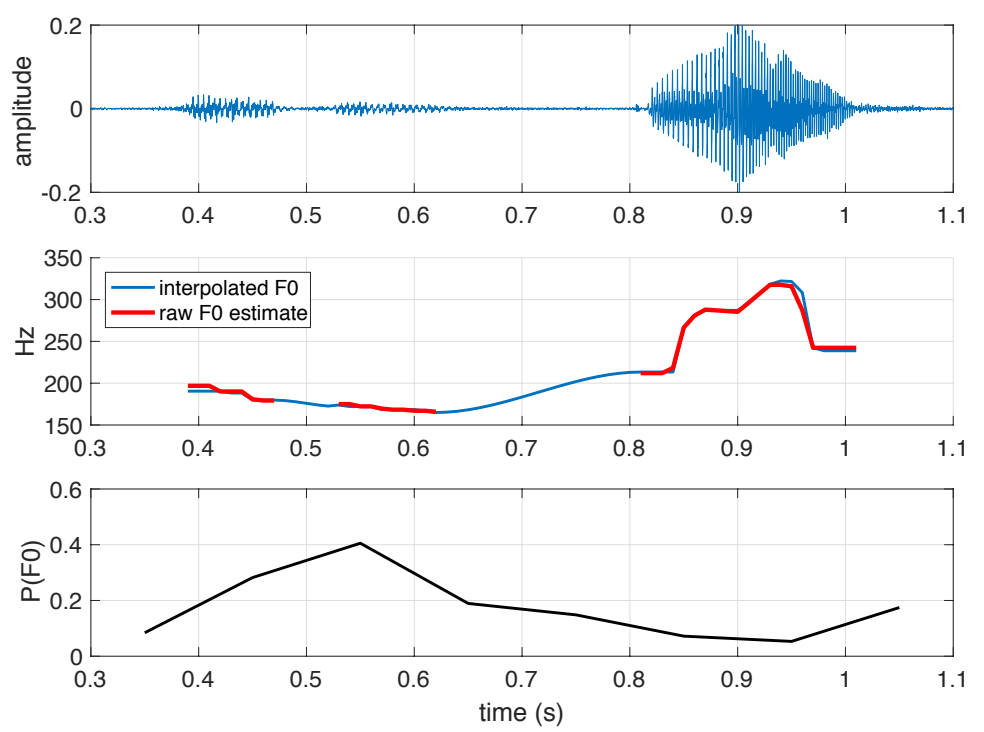

Figure 4: An example utterance "What is this?" with corresponding speech waveform at the top, the original and interpolated F0 contour in the middle, and the resulting F0 likelihood contour at the bottom (100-ms uniform frames with MOCM model). In this case, the initial falling pitch is considered as relatively predictable by the model. However, the uncertainty increases from the unvoiced frication to the sudden large and non-monotonic pitch change during the sentence-final stressed word "this".

After the training, segment-by-segment estimation of F0 likelihoods on the remaining held-out utterances was carried out using Eq. (1) or the LSTM network. The procedure was repeated in a 10-fold manner until all utterances had been used in the test set. The allocation of utterances into training and testing sets was purely random, and therefore both contained speech from the same 11 unique talkers. We decided not to use speaker-specific models for F0 due to the modest number of utterances per talker

\footnotetext{
${ }^{3}$ In order to use full utterances, the number of included ADS utterances was always $N-1$ where $N$ would have led to more ADS than IDS speech in seconds.
} 
that would have caused data sparsity issues in the model estimation. As a result, the obtained probability estimates describe how expected is the F0 behavior in the given context given a preceding exposure to a large number of F0 trajectories from both speaking styles, low probability reflecting unexpected and thereby potentially attention capturing intonation.

Note that the choice of $Q$, the number of quantization categories for the F0 shapes, contains an inherent tradeoff between the resolution of the F0 trajectory modeling and the amount of data required for model estimation. Although there is no a priori reason to consider any $Q$ specifically favoring IDS or ADS due to the z-score normalization of all F0 values, we wanted to minimize the impact of $Q$ in our analyses. Therefore the simulations were conducted for $Q=6,12$, and 24 with segment-specific likelihood estimates averaged across all these runs. In addition, all likelihoods were averaged across five runs of the entire experiment to diminish any variation caused by random initialization of the k-means centroids, parameters of the LSTM network, or sampling of the utterances for the training and testing data. Fig. 4 shows an example of an IDS utterance with its F0 trajectory and the resulting probability contour that was then used in the data analysis (the next section).

\section{Data analysis}

Five utterance-level statistical descriptors, namely, the mean, $S D$, min, max, and range (max-min) were calculated for the F0 likelihoods across all segments in each utterance. In addition, the corresponding descriptors were extracted from normal (unnormalized) F0 estimates in $\mathrm{Hz}$ as a reference. Talker and style-specific (IDS vs ADS) means for the descriptors were then averaged across all the utterances from the given talkers. Before the main statistical analyses, the statistical descriptors for F0 likelihoods were corrected for the variable amount of matching training data for the specific speaker and speaking style in question. This was done by first fitting a speaker-independent linear regression model from the number of matching training samples to the statistical descriptors, and then subtracting the speaker- and stylespecific prediction from the original value, thereby decorrelating the measures with respect to the amount of matching training data.

In order to test differences between IDS and ADS, the normalized descriptors for F0 predictability and descriptors for the original F0 values were then compared between the IDS and ADS conditions using paired t-test with a significance level of $p<0.05$ (Holm-Bonferroni corrected for the ten comparisons and $d f=10$ for all reported stats). The analyses were conducted separately for LSTM and MOCM models and for the syllabic and fixed-frame temporal segments in order to explore the robustness of any observed effects towards the modeling and sampling considerations. 


\section{Results}

The first main finding from the analyses was that the MOCM and LSTM models performed very similarly across the conditions and having highly-correlated segment-by-segment likelihood outputs $(r=$ 0.978 for fixed-frame and $r=0.984$ for syllabic segments; rank correlation). In addition, the main findings from the syllabic and fixed-frame analyses were highly similar, MOCM model with syllabic segments having the best overall fit to the data (with mean likelihood $E\left\{P\left(q_{s} \mid\right.\right.$ context) $\}=0.333$ per frame ${ }^{4}$ ), followed by syllabic segment LSTM (0.311), uniform segment MOCM (0.291), and uniform segment LSTM (0.268). For conciseness, this section focuses on reporting the results for the syllabic MOCM model, as it can be considered as representative for the other variants as well, and, unless otherwise mentioned, with only minor differences in the reported effect sizes. The corresponding results for all four variants can be found in Appendix A.
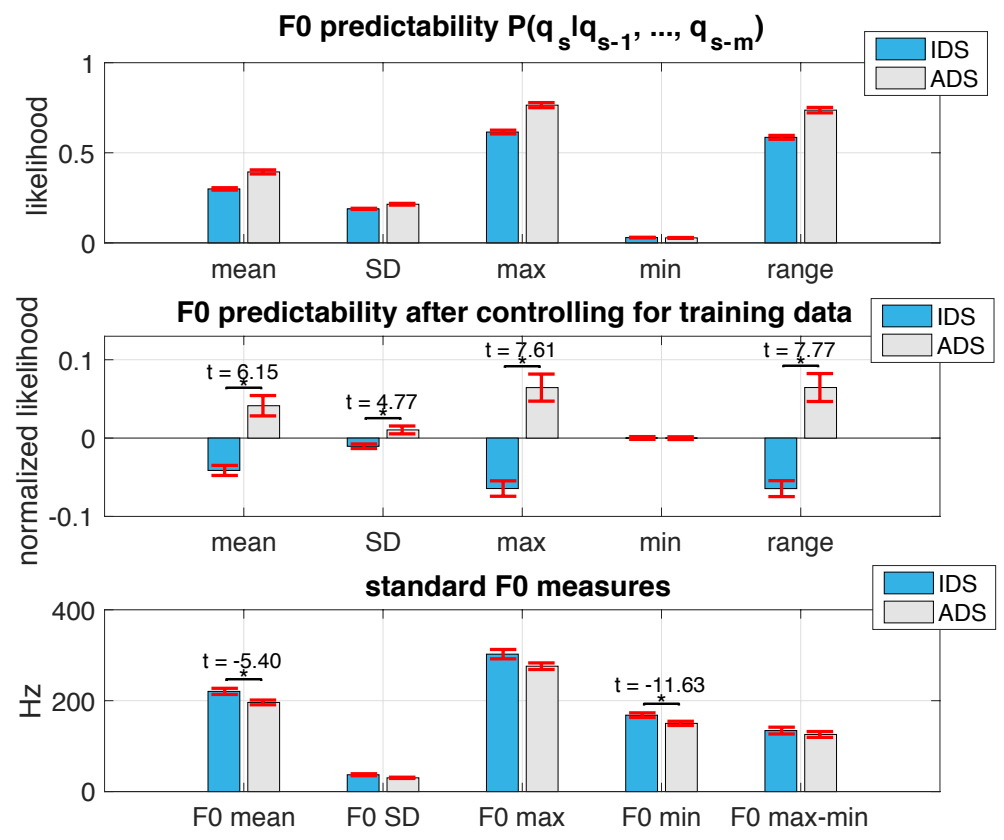

Figure 5: Results from the comparison between IDS and ADS in terms of F0 predictability and raw F0 values using the MOCM model with syllabic segments. Top: Utterance-level statistical descriptors of F0 predictability, averaged across all ADS/IDS utterances. Middle: F0 predictability after controlling for the amount of matching training data (speaker \& style) for each utterance. Bottom: Utterance-level descriptors of original F0 in $\mathrm{Hz}$, averaged across all utterances. Error bars denote \pm 1 SE across all talkers. Significant differences between IDS and ADS are denoted with asterisks and related t-values (paired t-test, $d f=10$, and using significance level $p<0.05$ with Holm-Bonferroni correction for the ten comparisons).

\footnotetext{
${ }^{4}$ Note that the number of frames for syllabic and uniform segments is not equal, and therefore likelihood per frame is reported instead of the typically used (log-)likelihood over all the data.
} 
Fig. 5 shows a summary of the MOCM syllabic segment results together with standard F0 descriptors, and where asterisks and t-statistics denote significant differences between IDS and ADS. As expected, the mean F0 frequencies of the 11 speakers are higher in IDS $(220.5 \mathrm{~Hz} \pm 22.4 \mathrm{~Hz})$ than in ADS (196.2 $\pm 17.2 \mathrm{~Hz})$. In addition, the minimum F0 is higher in IDS $(168.2 \pm 16.9 \mathrm{~Hz})$ than ADS $(150.1 \pm 15.3$ $\mathrm{Hz}$ ). The maximum F0 during utterances also seems to be higher in IDS (302.4 vs $275.9 \mathrm{~Hz}$ ) but does not reach significance after normalization for multiple comparisons, while the range and standard deviation of F0 are not different between IDS and ADS. In general, this pattern of baseline results is in line with the well-established finding that IDS has on average higher pitch than ADS.

As for the predictability, the mean predictability of F0 in IDS is significantly lower than in ADS $(t(10)=6.15$, Cohen's $d=3.89)$. In addition, the standard deviation of utterance-level F0 likelihoods is lower in IDS $(t(10)=4.77, d=3.01)$, as are the maximum $(t(10)=7.61, d=4.82)$ and range $(t(10)=$ 7.77, $d=4.91$ ) of the likelihoods. The same pattern of results is observed for all four variants of the model (Appendix A). The results therefore support our primary hypothesis that predictability of IDS intonation is different from ADS, and, specifically, IDS predictability is lower than that of ADS.

\subsection{Temporal analysis}

We were also interested in how the predictability of the intonation changes as a function of position in the utterances, and whether the differences in IDS and ADS would be specific to certain position(s). Since the data consist of utterances of various lengths with ADS utterances having substantially larger average duration, the analysis was conducted separately for short and long utterances using a division that ensured that several utterances from each talker ended up in both categories. In practice, we determined the set of short utterances by finding cutoff values for the maximum number of syllables in IDS and ADS utterances such that the style-specific distributions of utterance lengths (in syllables) were not statistically different from each other. We also required the total number of tokens in each length/style-group to be above 100. As a result, ADS utterances with 11 or less automatically detected syllables and IDS utterances with $\leq 13$ syllables were considered as $\operatorname{short~}(N=567$ IDS with a mean length of $M=6.7, \mathrm{SD}=3.18$ syllables, and $N=116$ ADS with $M=7.1, \mathrm{SD}=2.7$ syllables; difference not significant: $t(681)=1.43, p=0.152$, unpaired t-test). All other utterances were considered as long $(N$ $=106$ IDS with $M=18.2$ syllables, $N=281$ ADS with $M=26.1$ syllables; ADS utterances significantly longer). Even though the resulting short category still consists of relatively long utterances, considering 
typical IDS, cross-style comparison of shorter utterances was not possible due to the relatively few short ADS utterances in the ManyBabies corpus.

Figs. 6 and 7 show the F0 predictability measures as a function of the relative position in the utterances together with the corresponding average F0 contours. The reported values are based on resampling the varying-length F0 likelihood contours into ten bins and then averaging the results across all data (i.e., contours of different length tokens within the same length- and style-group were stretched or shrunk to have an equal length of 10 segments to enable the comparison). When looking at the trend of predictability across the duration of the short utterances, it appears that the predictability of IDS intonation first increases and then decreases towards the end of the utterances. This is also confirmed by correlational analysis where the probability increases during the first half of the utterance $(r=0.953, p=$ 0.012; linear correlation) and decreases during the last half $(r=-0.888, p=0.044)$. In ADS, the first half shows an increasing trend $(r=0.950, p=0.014)$ whereas the latter half does not exhibit any systematic decrease. An exception is the last segment that largely corresponds to the interpolated transition from utterance-final voicing to the first (discarded) segment without voicing. The difference between IDS and ADS towards utterance endings is also visible in the average z-score normalized $\log (\mathrm{F} 0)$ contour (Fig. 6, bottom panel): IDS tends to have on average higher F0 near the end of the utterances, whereas ADS has higher average normalized pitch in the middle sections of the utterances.

In long utterances (Fig. 7), the shape of the predictability contours is highly similar for IDS and ADS, both first increasing during the first half $(r=0.966$ and $r=0.991$ for IDS and ADS, respectively; $p<0.05)$ and then staying at a stable level except for the final transition to silence. The lack of any substantial local variations in the contours is expected, as the variance in the linguistic content and number of words in such long utterances is large, diminishing any potential effects of temporally localized prominent words in individual utterances. This is also reflected in the normalized F0 contours where the overall intonation contour is similar for long IDS and ADS utterances. 


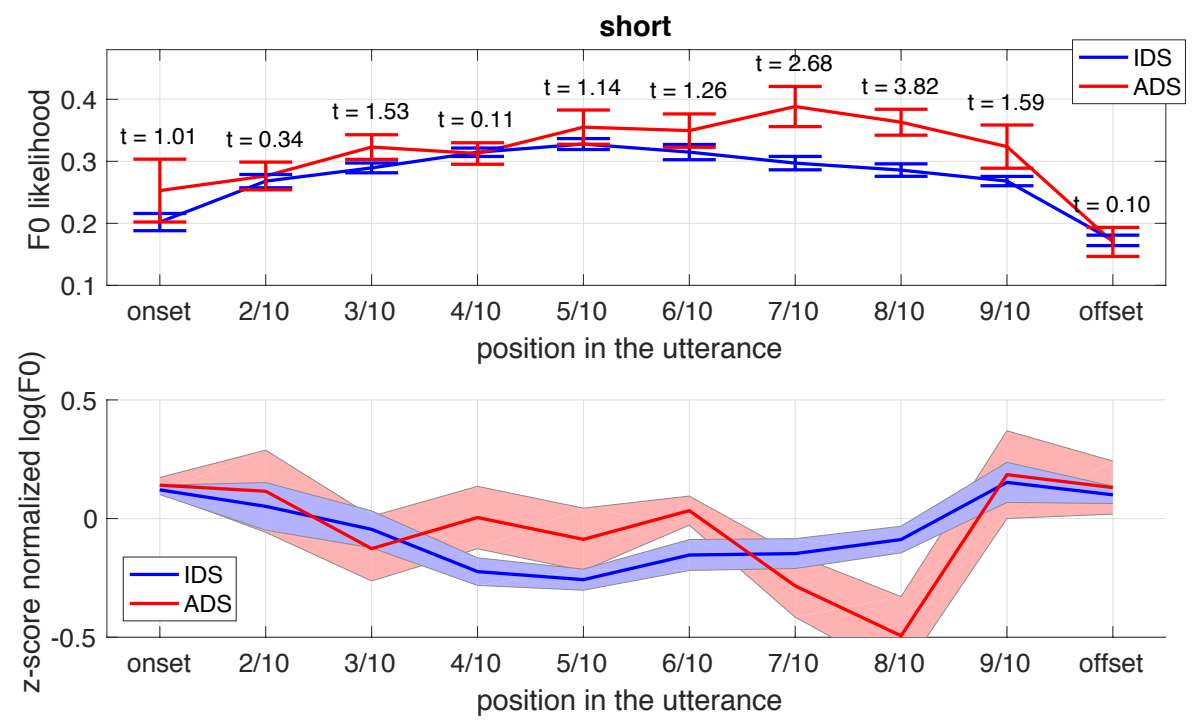

Figure 6: Top: mean predictability of IDS (blue line) and ADS (red line) F0 at different temporal positions in short utterances, measured across the 11 subjects (MOCM with syllabic frames). Bottom: Average z-score normalized $\log (\mathrm{F} 0)$-contours for the two speaking styles. Error bars or shading denote standard error of the mean.

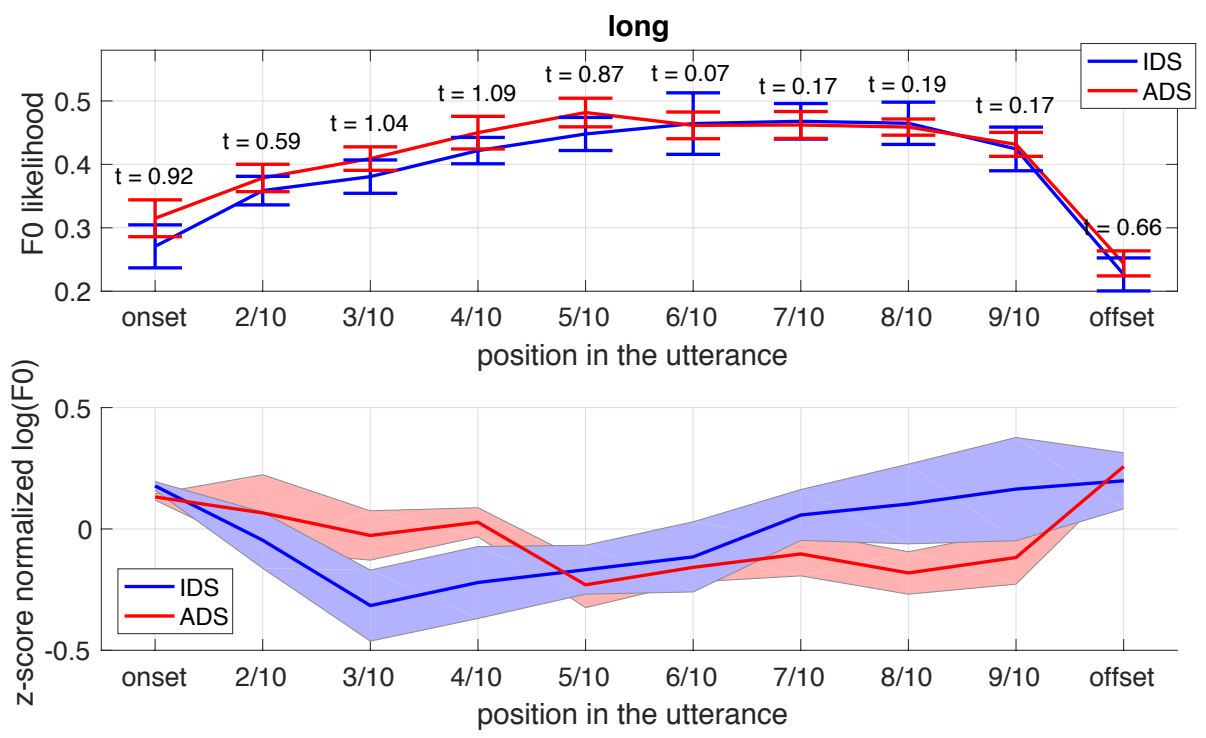

Figure 7: Top: mean predictability of IDS (blue line) and ADS (red line) F0 at different temporal positions in long utterances, measured across the 11 subjects (MOCM with syllabic frames). Bottom: Average z-score normalized $\log (\mathrm{F} 0)$-contours for the two speaking styles. Error bars or shading denote standard error of the mean.

Since short utterances are especially typical for IDS, we were also interested in whether the utterance length is related to the overall degree of surprisal in intonation. Fig. 8 shows the correlation between the number of syllables in IDS utterances and the mean, SD, max, and range of the F0 likelihoods during the same utterances. The same analysis is also shown for the raw F0 in Hz. According to the present 
statistical modeling approach, intonation in short utterances is harder to predict than in long utterances, even though the mean of standard F0 does not substantially depend on the utterance length. Overall, utterance length in syllables explains approximately $33 \%$ of the variance in average predictability. In addition, longer utterances tend to have more variable likelihood contours and slightly larger variance for F0 (in Hz) as well. The dependency of range and maximum descriptors on utterance length is not surprising, as longer utterances are more likely to exhibit extreme values on both absolute and probabilistic scales.
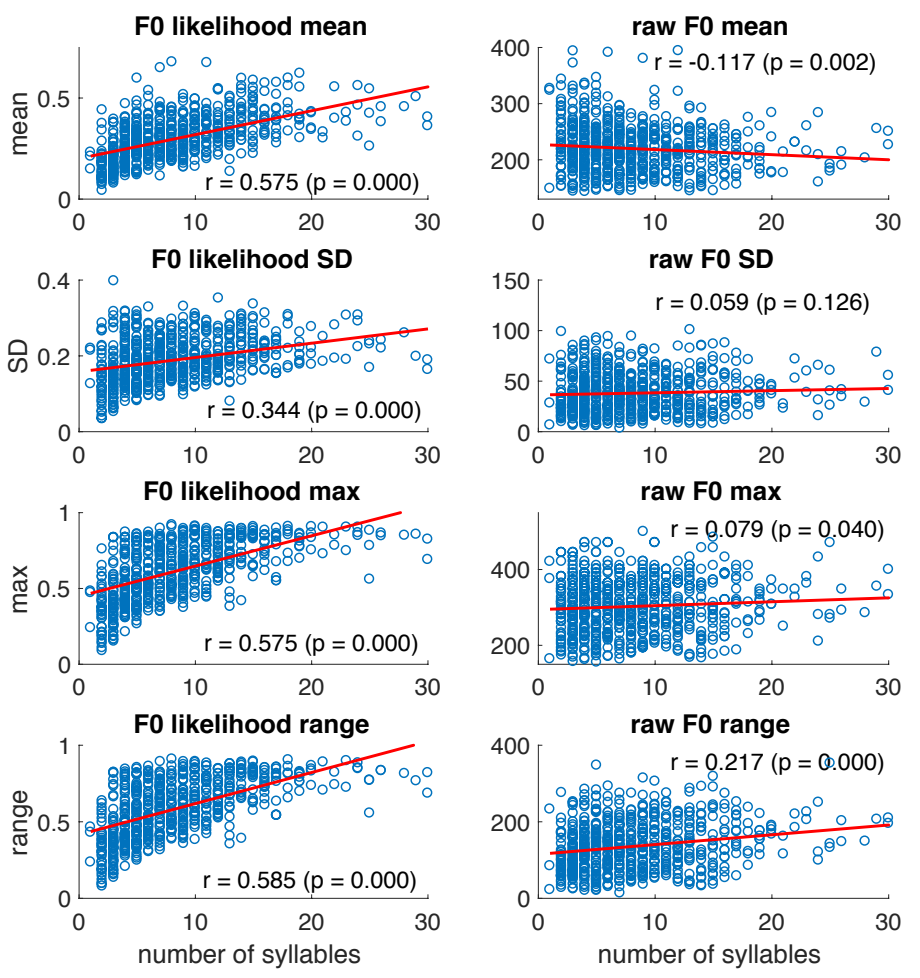

Figure 8: Linear correlation between IDS utterance length (in syllables) and mean, SD, max and range descriptors of F0 likelihoods (left) and F0 in $\mathrm{Hz}$ (right).

To see whether the correlation between predictability and utterance length depended on the position within the utterances, the analysis was also conducted separately for each of the time bins in the resampled likelihood trajectories (cf. Figs. 6 and 7) and the result is shown in Fig. 9. The main finding from the analysis is that F0 predictability at the middle- and end-sections of IDS utterances is much more dependent on the utterance length than those in ADS, IDS utterance endings becoming more predictable as the number of syllables ( $\sim$ words) in the utterance increases. Since the average predictability of IDS is still lower than ADS, this further illustrates that utterance-final intonation is 
especially surprising for short IDS utterances and the differences decrease with increasing utterance length (see also Figs. 6 and 7).

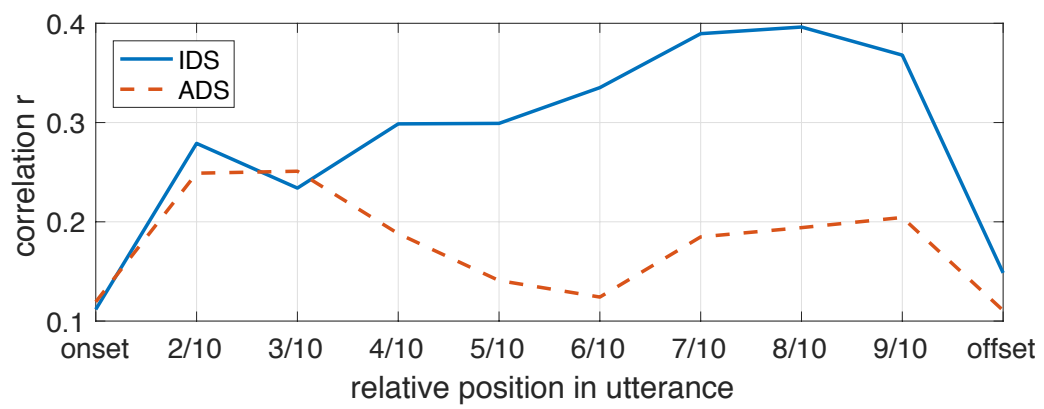

Figure 9: Correlation between utterance length (in syllables) and mean F0 predictability as a function of the relative temporal position in the utterances. Higher $r$ means that predictability at that position increases more with utterance length (see also Fig. 8, top left panel).

Importantly, the temporal analyses reveal that the differences in IDS and ADS predictability are not simply caused by the shorter average IDS utterance duration. In principle, predictions for F0 during the first segments (syllables) of each utterance can be assumed to be more difficult than later in the utterance simply because there is little or no preceding F0 context to rely on. If the IDS utterances are shorter on average, the relative proportion of these "difficult" segments becomes larger, and therefore this could lower the mean predictability of IDS in the data in contrast to ADS. However, as Figs. 6-9 demonstrate, the differences between IDS and ADS are not specific to utterance onsets, but show up as a systematic difference in the latter halves of short utterances. This rules out the possibility that utterance duration or any duration-related processing artifact would be solely responsible for the observed differences in IDS and ADS. The observed pattern of results also fits well with the empirical finding where novel content words tend to be located at utterance-final positions in IDS (Aslin, Woodward, LaMendola \& Bever, 1996; Fernald \& Mazzie, 1991), which may be helpful for word learning. 


\subsection{Sensitivity to proportion of IDS in language input}
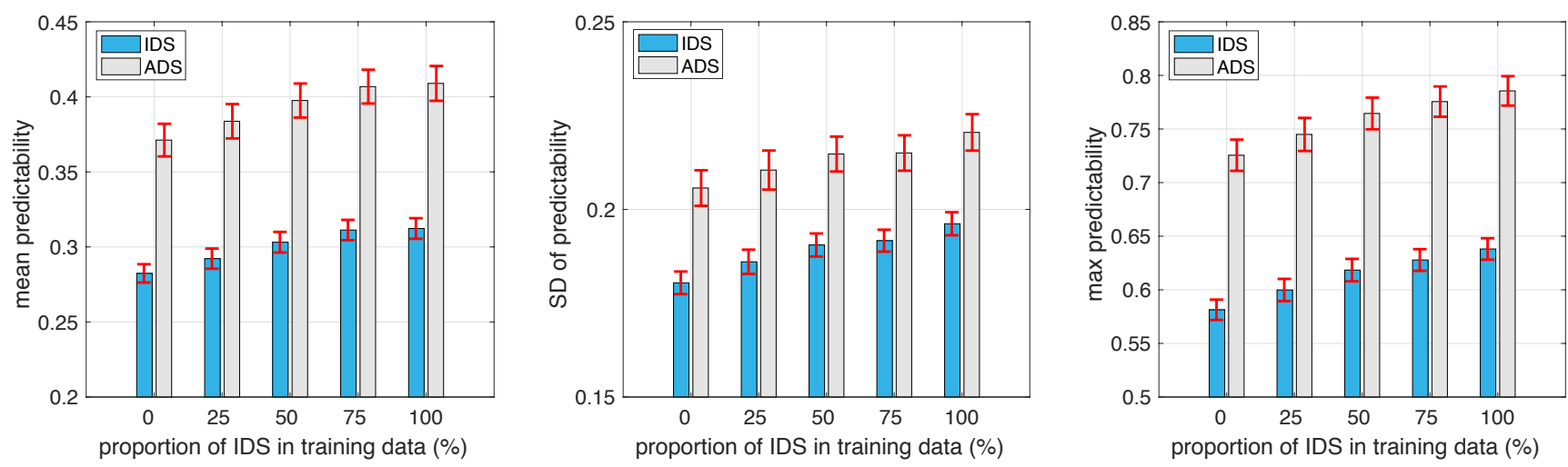

Figure 10: Mean, SD and max utterance-level predictability of F0 contours for IDS and ADS speech for different proportions of IDS in the training data with the rest consisting of ADS speech. The results are shown for MOCM temporal model using syllabic segmentation. The total amount of training data (in seconds) is the same for all proportions. Error bars denote $\pm 1 \mathrm{SE}$ across all talkers.

It is unlikely that infants hear exactly equal amount of ADS and IDS in their natural environments, and therefore the so-far used balanced training setup is not necessarily representative of prosodic learning and perception in the wild. In reality, the proportion of IDS from all speech input to infants can depend on factors such as cultural environment, socioeconomic status, age of the child, or other family-specific factors, reports ranging from $20 \%$ to $70 \%$ of all speech in the vicinity being directed to the child (e.g., Shneidman \& Goldin-Meadow, 2012; Weisleder \& Fernald, 2013; Cristia et al., in press). It was therefore of interest whether the present findings would hold when the proportion of IDS in the language input varies from the previously used equal exposure to IDS and ADS. To study this, we re-ran our main simulations by setting the amount of IDS speech to $0 \%, 25 \%, 50 \%, 75 \%$ or $100 \%$ of the training data (measured in seconds). The total amount of training data was fixed to 20.3 minutes corresponding to all IDS available in the corpus ${ }^{5}$, and we did not apply any speaker- and style-dependent normalization of the likelihoods as was previously done in the main analyses. Fig. 10 shows the results for the main descriptors of interest (mean, SD, and max of F0 likelihood), using MOCM model with syllabic segments. Since the results were again qualitatively the same for LSTM and MOCM, and for fixedframe and syllable-frame segmentation, the other variants are not shown separately.

\footnotetext{
${ }^{5}$ Since there was more ADS than IDS in the corpus, this was done to ensure that overall amount of training data does not vary between different proportions of IDS in the training set.
} 
There are two key findings to be observed in Fig. 10. First, the IDS intonation is less predictable independently of the proportion of IDS in the training data, confirming that the effect is not critically dependent on the previous assumption of balanced IDS and ADS exposure. Secondly, the predictability of both IDS and ADS is higher when there is more IDS in the training data. That is, hearing IDS seems to be also more beneficial for statistical learning of ADS intonation contours than hearing speech in adult-directed speaking style, when learning is measured in terms of capability to predict intonation in novel sentences. What this somewhat counterintuitive finding suggests is that the more variable temporal dynamics of IDS not only contain just some abstract "information" in terms of average surprisal (self information defined as $I=-\log (p)$ ), which could be simply due to highly volatile F0 behavior that is difficult to learn, but would still align with the predictability-based theory for attentional orientation. Instead, the higher information density of IDS also translates into faster learning of intonation contours, which, technically speaking, means that the exposure to IDS has higher information gain (reduction in predictive uncertainty) for the present statistical learning models. This is concrete modeling support for the idea that the more variable IDS speaking style may boost prosodic learning in infants. In contrast, the previous studies on the potential benefits of higher acoustic variability of IDS in phonetic learning have been more mixed (e.g., de Boer \& Kuhl, 2003; Kirchhoff \& Schimmel, 2005; McMurray et al., 2013; Martin et al., 2015).

This control experiment also suggests that the lower predictability of IDS F0 is not simply due to larger quantization errors of the F0 parameters or larger errors in F0 estimation, as such style-specific biases should not translate into better learning of ADS trajectories. However, it is still possible that the slightly more variable F0 parameters (Fig. 3, right) in IDS in comparison to ADS acts as an implicit regularizer for the statistical models, preventing over-fitting to the training data, and thereby leading to better generalization towards novel utterances in both speaking styles. To make sure that this was not the case, we also re-ran the experiment using only 3 iterations of EM-algorithm in the MOCM-model estimation (default $=30$ ), stopping learning well before the convergence of the model parameters. This did not cause qualitative changes in the results, but led to lower overall predictability for both IDS and ADS due to the less accurate models. This suggests that model over-fitting to ADS but not to IDS was not behind the pattern of the results observed.

\subsection{Further analyses}

As a follow-up validation of the main findings, we also ran binary logistic regression to classify all the individual utterances into IDS or ADS classes using the utterance-level descriptors for probabilities and 
raw F0 values as features (using SPSS version 24.0, IBM Corp., Armonk, NY). MOCM with syllabic segments was used to extract the predictability descriptors, and the regression likelihood ratio was used as the criterion for forward stepwise feature selection. The resulting model achieved IDS/ADS utterance classification accuracy of $76.8 \%$ using a final set of six features: SD of likelihood (Wald statistic = 55.57, $p<0.001 ; d f=1$ for all features), mean likelihood $(W=51.96, p<0.001)$, likelihood range $(W=$ $39.48, p<0.001)$, together with mean of original F0 $(W=9.775, p=0.002)$, F0 SD $(W=27.64, p<$ $0.001)$, and $\mathrm{F} 0$ range $(W=3.00, p=0.083)$. Utterance classification using only the five probability descriptors led to $73.9 \%$ accuracy while the corresponding performance for normal F0 descriptors was $65.5 \%$. Since computation of the probability descriptors never made any use of the knowledge of the underlying style of speech, this indicates that the predictability differences in IDS and ADS do not simply appear as aggregate measures across a large number of utterances, but can be also used as a cue to classify individual utterances into ADS or IDS.

In addition, a subset of the utterances $(N=111)$ used in the present study had been previously rated by several Mechanical Turk workers for their IDS-likeness in the context of the ManyBabies study and for which the ratings were publicly available. In the listening test, the subjects heard low-pass filtered versions of the recordings and were asked to rate their IDS-likeness using a 7-point Likert scale $(1=\mathrm{ADS}, 7$ = IDS). This rated subset only includes utterances that also meet a number of other exclusion criteria based on the same listening test, including clear IDS- or ADS-likeness, low noise level, high naturalness, and consisting of standard English (see The ManyBabies Consortium, 2017, for details). Since we were interested how absolute vs. probabilistic properties of F0 are reflected in the ratings, the correlations between all the utterance-level F0 descriptors and the human IDS-likeness ratings were computed and are reported in Table 1.

Table 1: Rank correlation between human ratings of IDS-likeness of utterances and the corresponding utterancelevel measures of F0 in $\mathrm{Hz}$ (left) and its predictability (right). Significant correlations are denoted with asterisks * (Holm-Bonferroni correction for 11 comparisons at $\mathrm{p}<0.05$ ).

\begin{tabular}{|l|c|c|l|c|c|}
\hline descriptor & $\boldsymbol{r}$ & $\boldsymbol{p}$ & descriptor & $\boldsymbol{r}$ & $\boldsymbol{p}$ \\
\hline F0 mean & 0.513 & $<0.001^{*}$ & $\mathrm{P}(\mathrm{F0})$ mean & -0.316 & $<0.001^{*}$ \\
\hline F0 SD & 0.457 & $<0.001^{*}$ & $\mathrm{P}(\mathrm{F0})$ SD & -0.224 & $0.001^{*}$ \\
\hline F0 max & 0.483 & $<0.001^{*}$ & $\mathrm{P}(\mathrm{F0})$ max & -0.427 & $<0.001^{*}$ \\
\hline F0 min & 0.361 & $<0.001^{*}$ & $\mathrm{P}(\mathrm{F0})$ min & 0.295 & $0.002^{*}$ \\
\hline F0 range & 0.378 & $<0.001^{*}$ & $\mathrm{P}(\mathrm{F0})$ range & -0.467 & $<0.001^{*}$ \\
\hline log(duration) & -0.611 & $<0.001^{*}$ & & & \\
\hline
\end{tabular}


Even though F0 predictability was a more reliable cue for classification utterances into IDS and ADS in the full pool of utterances, the subjective ratings of IDS-likeness seem to be slightly more correlated with the physical properties such as the mean, maximum, and variance of F0 during the utterances. However, the rated utterances only consist of a carefully selected subset of all tokens, and the listeners' task (“How representative of IDS is this?") was also somewhat different from the classification task ("Is this IDS or ADS?"). A closer analysis suggests that the difference is largely explained by the former factor. When only the 111 human-rated utterances were submitted for binary logistic classification of the speaking style, the classification accuracy was $82.0 \%$ for the raw F0 utterance-level descriptors, $73.0 \%$ for the probability-based descriptors, and $86.5 \%$ for their combination, reversing the order of feature importance from the full pool of utterances. This shows that the ManyBabies screening procedure for "prototypical" IDS and ADS favors salient F0 behavior (utterance max and mean especially), which is not surprising as high F0 is often considered as a part of the prototypical IDS. However, what is unexpected is that the predictability as a cue to speaking style becomes less informative in this prototypical subset in comparison to the full pool of utterances $(65.5 \%$ vs $73.9 \%$ classification accuracies, respectively), indicating that the F0 (in Hz) and its predictability represent at least partially different aspects of the signal. Stepwise forward selection in linear regression from the 11 features in Table 1 to the 7-point human ratings also results in a model that only uses the maximum F0 and log-duration of the utterances as the explanatory variables $\left(r^{2}=0.552, \mathrm{SD}_{\text {error }}=1.05\right)$. Based on the current data, it is impossible to conclude whether this is because 1) subjective listening-based ratings for IDS-likeness are biased towards perceptually accessible properties, such as F0 magnitude and range instead of capturing the full extent of IDS properties that might be relevant for its functional role ${ }^{6}$, or 2) whether F0 magnitude simply becomes more systematic at the extremes of the IDS/ADS continuum ${ }^{7}$ while the probabilistic properties stay much more constant during infant-caregiver or adult-adult communication scenarios. In principle, the latter might be also caused by z-score normalization of the F0 trajectories for our present modeling purposes. However, we repeated the analysis without z-score normalization of F0 and without any qualitative changes in the findings. Still, combination of the absolute F0 properties with predictability measures reaches notably higher classification rate for the

\footnotetext{
${ }^{6}$ Remember that the primary aspects of interest here are the attention-capturing properties of IDS versus ADS. Since such information selection processes by definition operate at a pre-attentional level, there are no guarantees that subjective perceptual evaluation of stimulus IDS-likeness is based on the same properties of the signal that drive attentional focus during real-time comprehension.

${ }^{7}$ One could argue that the idea of selecting or rating "the most representative" IDS or ADS utterances from a larger pool of recordings is an ill-defined task. In a general sense, any naturalistic speech directed at infants should be considered as representative IDS and any speech directed at adults as representative ADS, even if acoustic or perceptual features of IDS deviate from ADS to varying degrees in different utterances.
} 
speaking style also in this carefully selected subset of data. This indicates the complementarity of the two types of intonation representations also in separating prototypical IDS from ADS.

Finally, we tested whether there were any differences in utterance-level F0 predictability or frequency descriptors between the sentences containing words referring to the presumed familiar versus unfamiliar objects (see Section 2). However, no significant effects were observed after controlling for multiple comparisons. In contrast, there was a systematic difference in the predictability between IDS and ADS utterances within each of the three naming categories (familiar object, unfamiliar object, no object naming in the utterance) with average IDS predictability being always lower $(p<0.001$, unpaired $\mathrm{t}$-test for all the tokens from the given naming category). This was somewhat surprising, considering that unfamiliar object words should be more relevant learning targets for the children, and hence one might expect that caregivers would highlight such utterances over those with familiar content words. Potential explanations for the lack of any substantial differences in aggregate F0 behavior were therefore investigated by manually listening to the utterances. Although anecdotal, this revealed that there was little perceptual difference in intonation between utterances referring to familiar and unfamiliar objects. We also found that caregivers often highlighted some other aspects of the sentences in the context of familiar objects. For instance, "A green block.", "Drink from the cup, it's almost like a bottle!", or “What do you do with the cup?" (prominent words with bold-face font) all contain a presumably familiar word (cup or block), but intonation is used to highlight additional information such as object properties, potential actions, or relationships to other objects instead of the object itself. Based on this, we hypothesized that while discussing familiar objects, mothers may have been more likely to choose alternative topics to be the focus of utterance while preserving the overall predictability of the intonation as compared with utterances discussing unfamiliar words.

To test this hypothesis, the first author manually segmented all keywords from the IDS recordings and the comparison was repeated for the likelihood trajectories and original F0 values measured only during the keywords. Even in this case, no statistically significant differences between familiar and unfamiliar objects were observed. This suggests that the mothers were not altering their speaking style based on object familiarity. However, these findings should be considered with caution due to a number of factors: First of all, the names of the familiar and unfamiliar objects were not phonetically balanced and the number of naming occurrences per object was not balanced across different mothers, potentially biasing the results. In addition, the infants were 4-8 months old, but even 
the presumably familiar words are actually understood ${ }^{8}$ by most infants only around 13 months of age and produced much later (CDI lexical norms; Dale \& Fenson, 1996; Fenson et al., 2007). In other words, it is possible that the mothers were treating both types of objects as potential learning targets for their children, consciously or unconsciously, and despite the intended recording scenario manipulation.

\subsection{Other control experiments}

We also repeated the main analyses shown in Fig. 5 using a linear instead of a $2^{\text {nd }}$-order model for the F0 contours (i.e., encoding only the direction and rate of change in F0 during each segment). This replicated all the main findings. In addition, the predictability difference is not simply due to a larger quantization error for IDS parameters, since the reported pattern of results persisted also if only the IDS data were used for the k-means clustering, leading to a lower quantization error (RMSE) for the IDS F0 trajectories. Together with the use of scale-free F0 representation, speaker-specific matched data normalization in the analyses, and analyses conducted in Sections 5.2 and 5.3, the tests support the finding that the intonation contours in IDS are less predictable over time than in ADS, at least for the present data and modeling approach in question.

\section{Discussion and conclusions}

Since stimulus-driven attention is related to the predictability of the stimulus (see the introduction), it is of interest whether the predictability characteristics of IDS prosody would somehow differ from ADS, as one proposed role of IDS is to increase infants' attentiveness to the speech stream. To shed light on this issue, the present study aimed to test whether the exaggerated intonation in IDS also translates into less predictable prosody over time when compared to normal adult-directed speech when the learner has previous experience from both speaking styles. The present results support this idea, even when the actual mean and variance of F0 values in the predictive analysis were normalized between the IDS and ADS utterances. More detailed analyses also confirmed that the difference between the speaking styles cannot be explained solely by the inherent differences in utterance durations, and that the lower predictability of IDS intonation does not critically depend on the proportion of IDS in the total experience with speech. However, prosodic predictability and utterance duration were still found to be coupled, short utterances being less predictable in general. We also found that short IDS utterances (i.e., the majority of the IDS data) have less predictable intonation contours towards the ends of the utterances

${ }^{8}$ To be precise, CDI receptive lexicon norms describe the age when the caregivers start to believe that their infants understand the words. However, it is likely the caregiver's beliefs, not the infant's actual underlying knowledge, that would drive word familiarity effects in caregiver speech. 
than ADS utterances of the same length, matching with the typical position of content words at utterance-final positions. Taken together, these findings provide initial support for the idea that one reason why IDS is attentionally more attractive could be because it is more surprising. This would allow IDS to tap to generic cognitive mechanisms for orienting towards aspects of the environment that violate prior predictions based on earlier experience.

However, one key question is whether the IDS predictability corresponds to a level of uncertainty that is optimal for capturing infant attention, as characterized by the so-called Goldilockseffect (Kidd et al., 2012, 2014). What the Goldilocks-effect says is that infants prefer to attend to less likely stimuli over highly predictable stimuli, as long as the predictability is not too low. This suggests that stimulus-driven attention operates in a region where there is sufficient structural regularity to differentiate relative probabilities of different patterns given the current predictive context. In computational terms, a prerequisite for evaluating the information value of different sensory inputs (and hence prioritizing their processing) is that the perceiver's brain must be able to compare each stimulus against an existing predictive distribution for the input (cf. also Friston, 2010). This is because the highest potential information gain ${ }^{9}$ is generally associated with inputs that violate well-established prior predictions, not just any stimuli that are difficult to predict. If the predictive context preceding a stimulus is always highly complicated compared to the number of examples available to the learner, it is also difficult for the subject to learn predictive dependencies for the situation. As a result, it is also challenging to evaluate the likelihood of a stimulus in a highly volatile context.

The main challenge in relating the present findings to the existing studies on Goldilocks-effect is that the earlier works have used stimuli that can be considered as categorical, such as sequences of pictures in the original study of Kidd et al. (2012) or sequences of different natural sounds in Kidd et al. (2014). In these cases, the probabilistic structure of the stimuli can be explicitly defined using discrete mathematics and the structure of the predictive contexts can be also measured accordingly. In contrast, F0 trajectories of speech are inherently continuous-valued and temporally contiguous. This means that there is no unanimous way to determine whether two F0 trajectories or their parts are the same or different. Even if a perceptual auditory model could be used to define just-noticeable differences in F0 frequencies, it is substantially more difficult to incorporate the model with the type of representations and metrics infants might use to analyze, learn, and distinguish intonation patterns at different stages of their language development. For instance, we would have to know how factors such as absolute vs.

\footnotetext{
${ }^{9}$ For a mathematical treatment, see Shannon (1948) or the concepts of Kullback-Leibler divergence and expected selfinformation.
} 
relative F0, rate of F0 change, or potential speaker-normalization mechanisms might impact perceptual judgments of prosodic similarity in infants whose auditory system is still undergoing developmental changes.

The present study has circumvented the issue of non-categorical input by quantizing the F0 values in frequency and time, and then investigating the differences between IDS and ADS across different resolutions in both domains. In addition, we z-score normalized logarithmic F0 values for all utterances to ensure that any observed differences arise from temporal predictability of intonation instead of inherent differences in F0 range or the quantization errors that would result from such differences. The drawback of this approach is that it is not possible to define an "absolute" measure of probability for the observed intonation patterns that would allow us to relate the current findings to the typical complexity scales observed in Goldilocks-studies. Instead, our approach allowed us to probe the relative predictability of IDS and ADS using as comparable settings as possible. The absolute likelihood values reported for the predictive models are dependent on the choice of the analysis resolution for time and frequency, lower F0 resolution and shorter temporal distances leading to higher average likelihoods. However, the experiment described in Section 5.2 shows that listening to IDS leads to better prosodic learning outcomes than listening to the equal amount of ADS, other factors fixed, and that learning from the more variable IDS also generalizes to ADS intonation patterns. This suggests that the lower predictability of IDS is not just due to highly random input with little functional value (i.e., too lowprobability non-attractive stimuli in the context of Goldilocks-effect), but because the increased variability in IDS actually carries more information per time unit on how intonation can behave in speech. However, further experiments on more extensive and ecologically valid data are required to confirm these initial findings.

Overall, the present study cannot answer the question whether infants are actually learning regularities at the level of prosodic acoustic features and whether complexity of these statistical patterns is responsible or suitable for attentional capture. Instead, the study simply provides the first evidence that statistical properties of IDS differ from ADS in a manner that is compatible with the idea of stimulus-driven attention being oriented towards surprising aspects of the environment. That is, more attention would get directed at sensory events that have higher information gain with respect to the current model of the perceptual world (Zhang et al., 2008; Itti \& Baldi, 2009; Zarcone et al., 2016). In addition, low probability intonation patterns have been earlier linked to perceived prominence in speech (Kakouros \& Räsänen, 2016) similarly to low-probability words (e.g., Cole, Mo \& Hasegawa-Johnson, 2010). The current results therefore link informal descriptions of IDS as more salient or prominent (e.g., 
Garnica, 1977; Fernald et al., 1989; Soderstrom, 2007 and references therein) to measurable properties of the speech signal that are also related to learning from the input.

Importantly, the role of prosodic statistical learning in IDS is something that can be tested behaviorally in future experiments by carefully manipulating statistical properties of prosodic contours while controlling for other factors such as absolute magnitudes of acoustic prosodic features and lexical content of the stimuli. If the predictability hypothesis for attentional capture is correct, infant attention, as modulated by the predictability of the prosodic contours, should be separable from the presence of absolute physical cues such as F0 or energy peaks. However, this should only take place if the statistics of the prosody are not too simple or too complicated for the infants to capture during a familiarization stage (see also Kakouros, Salminen \& Räsänen, 2018, for a related study on adult subjects where statistical predictability of intonation was causally related to the subjective impression of sentence prominence in speech).

In addition, it was found that human adult ratings of IDS-likeness on a subset of the studied utterances could be largely explained in terms of F0 maximum frequency and duration of the utterances. This was observed even though temporal predictability of the intonation was generally found to be an efficient cue for automatically classifying speech into IDS and ADS. In fact, the predictability-based account to prominence and auditory attention in speech implies that the attention capturing properties of speech segments do not necessarily go hand-in-hand with specific acoustic features such as high or quickly changing pitch, as long as these features are to be expected in the given context. More specifically, it can be argued that the perceptual system should allocate processing resources to the aspects of the input that are not yet predicted by the brain independently of the absolute properties of the input. In the context of speech, this means that the talker can control the listener's attention by freely using non-canonical prosodic forms or words without changing the semantics of the utterance, leading to the subjective impression of emphasis also referred to as prominence (Kakouros et al., 2018). The present study suggests that caregivers may (implicitly) utilize a similar strategy to maintain infants' attention on the speech stream, either overall, or by highlighting certain segments of the speech stream. In contrast, highly predictable inputs, by definition, have low information value and are therefore low priority targets for sensing and learning even if they have large magnitudes on some scale such as loudness or pitch (e.g., Kakouros et al., 2018; see also, e.g., Friston \& Kiebel, 2009). However, the surface properties of speech could be more accessible to conscious analysis of the speaking style than the actual attention triggering properties of the signal that, by definition, should operate pre-attentively. In addition, acoustic cues still communicate other kinds of information, such as caregiver affection that 
is generally associated with IDS (see also below). These may partially explain why adult ratings of IDSlikeness seem to be biased towards superficial acoustic features.

Also, we want to emphasize that even if the present study focuses on the predictability at the level of prosody, the general idea of attention and learning being driven by (statistical) information seeking should not be considered as specific to prosody, but should take place at any level where statistical regularities are accessible to the listener. Until proven otherwise, it is parsimonious to assume that experience-based attentional preferences at the prosodic level, at the syllabic and lexical levels (e.g., Saffran et al., 1996), and between multiple representational levels such as acoustic words and their visual referents (e.g., Smith \& Yu, 2008) would be all based on the same fundamental cognitive mechanism for attending to information. If this is the case, then increased input variability could also have consequences to attentive processes during other aspects of language learning such as phonetic learning from IDS or learning from synthesized versus natural speech. Future research should therefore consider how learning from more variable input is potentially supported by increased attention to the input, and contrast this with the question of how the additional variability actually adds to the ecological usefulness of the learned representations. In the present study, the higher variability of IDS intonation also led to more useful models of intonation (Section 5.2), but this does not always have to be the case. For instance, McMurray et al. (2013) have suggested that the higher variability of voice onset times (VOTs) and formant frequencies in IDS may be counter-productive to phonetic learning from speech, as the variability does correspond to that observed in typical ADS (see also Kirchhoff \& Schimmel, 2005). However, this does not exclude the possibility that the increased variability at the segmental level may still support attentional processing of the input, suggesting that general representativeness of the input may sometimes become traded for increased attention towards the speech stream.

\subsection{Open questions and concluding remarks}

One largely untouched aspect of the work relates to the fact that intonation (and prosody in general) also plays other roles in speech beyond the hypothesized attentional control. For instance, suprasegmental features of speech are closely coupled to the lexical and syntactic properties of the language, and intonation is used to mark stress at the level of words and sentences or to create phonemic contrasts in tone languages. In addition, prosodic features such as F0, timing, and energy also carry information on the affective state of the talker (e.g., Banse \& Scherer, 1996; Schuller et al., 2011). One of the hypothesized roles of IDS is therefore to convey positive feedback and encouragement to the child (e.g., Fernald, 1989, Penman, Cross, Milgrom-Friedman \& Meares, 1983; Trainor, Austin \& Desjardins, 
2000; see also Spinelli et al., 2017, and references therein). This means that the attention-capturing prosodic contours cannot be produced independently of the literal messages, communicative intents, or underlying affective states. Instead, all the different roles of prosody must share the same acoustic signal available to the listener, who then decodes it back into its potential constituents. However, this does not have to mean that the different roles of prosody have to always compete in priority to shape the signal. For instance, it is known that sentence stress and prominence are related to the information and syntactic structure of the discourse (Calhoun, 2010; Shattuck-Hufnagel \& Turk, 1996) with new or otherwise important information receiving emphasis (also in intended contrasts such as "No, I went home" vs. "No, I went home"), thereby serving communicative intents. In addition, some evidence suggests that stressed or accentuated words are processed faster and more deeply (Cutler \& Foss, 1977; Sanford et al., 2006; Wang et al., 2011; Li \& Ren, 2012), implying that the prosodic emphasis alters the mode of semantic processing for the input.

Earlier research (Kakouros and Räsänen, 2016; Kakouros et al. 2018) has proposed that unpredictable prosody may actually cause an impression of prominence (similarly to unpredictable words; see Cole et al., 2010) by triggering attentional resources in exactly the same way as surprising IDS intonation is proposed to capture infant attention in the present study. Less likely intonation was also found to alter semantic processing of the prominent words, as measured by electrophysiological recordings (Kakouros et al. 2018). In the context of IDS, the apparent prominence (saliency) of the signal, the enhanced attentional capture, the prosodic highlighting of important words, and improved learning from the input (e.g., Song, Demuth \& Morgan, 2010; Ma et al., 2011; and Graf Estes \& Hurley, 2013) could be therefore all based on one and the same domain-general mechanism for selective information processing instead of multiple competing goals and processes (see also Kakouros et al., 2018, for a discussion). If the acoustic cues for such communicative intents also convey positive affect, thereby further encouraging the infant to engage in social interaction, all these factors would jointly serve the purpose of boosting language learning of the child. This also leads to the question whether there are in fact any "core" defining properties of IDS that would separate it from any speech with high effort for communicative success (in terms of intended information structure, listener attention, and high signal-to-noise ratio) while simultaneously conveying positive affect (cf. Singh et al., 2002) and taking the language skills of the listener into account (cf. Snow, 1977; Phillips, 1973; Stern, Spieker, Barnett \& MacKain, 1983), and if so, what is the functional role of these additional properties.

Despite being a highly relevant topic for language acquisition research, disentangling the different factors and mechanisms underlying IDS prosody and its perception is beyond the scope of the 
present study. The primary goal of this paper has been to bring forth a formal and testable hypothesis about the link between predictability and attention in IDS, and to initially study the hypothesis using a computational modeling approach. In addition, the purpose is to encourage other researchers in the field to work towards better understanding of the cognitive underpinnings of IDS and how it relates to learning and attention mechanisms of the human cognition, providing the community with an initial hypothesis to be discarded in favor of a stronger one. Overall, much more work is needed, including the need to replicate the present investigation using different modeling techniques and on more extensive speech data, and testing of the related hypotheses in actual behavioral experiments. In addition, the differences in IDS and ADS are not limited to intonation, and therefore aspects such as timing, utterance duration, intensity, and timbre should be investigated from the predictability point of view independently and in conjunction with F0. Finally, the potential disparity between acoustic and statistical cues that differentiate ADS from IDS and cues used by listeners to evaluate IDS-likeness of speech warrants further research, as it might inform us about any potential differences between what IDS does and how IDS sounds.

\section{Acknowledgments}

O.R. was funded by Academy of Finland projects no. 274479 and 312105 . S.K. was funded by Academy of Finland project no. 312490. M.S. is funded by a grant from Canada's Social Sciences and Humanities Research Council \#435-2015-0628. All MATLAB scripts for syllabification, mixed-order Markov chains, and the main experiments can be found at http://github.com/orasanen/IDS predictability. The original recordings are available from The ManyBabies Consortium at https://osf.io/re95x/. The authors would like to thank Michael C. Frank for his help with the ManyBabies data. An early version of this work was presented at the $39^{\text {th }}$ Annual Meeting of the Cognitive Science Society (CogSci-2017) at London, UK in July 2017.

\section{References}

Albin, D. D., \& Echols, C. H. (1996). Stressed and word-final syllables in infant-directed speech. Infant Behavior and Development, 19, 401-418.

Aslin, R., Woodward, J., LaMendola, N., \& Bever, T. (1996). Models of word segmentation in fluent maternal speech to infants. In Morgan J. \& Demuth K. (Eds.): Signal to Syntax. Bootstrapping from Speech to Grammar in Early Acquisition (pp. 117-134). Mahwah, N.J.: LEA. 
Aylett, M., \& Turk, A. (2004). The smooth signal redundancy hypothesis: a functional explanation for relationships between redundancy, prosodic prominence, and duration in spontaneous speech. Language and Speech, 47, 31-56.

Banse, R., \& Scherer, K. R. (1996). Acoustic profiles in vocal emotion expression. Journal of Personality and Social Psychology, 70, 614-636.

Bernstein Ratner, N. (1986). Durational cues which mark clause boundaries in mother-child speech. Journal of Phonetics, 14, 303-309.

Buxó-Lugo, A., \& Watson, D. G. (2016). Evidence for the influence of syntax on prosodic parsing. Journal of Memory and Language, 90, 1-13.

Calhoun, S. (2010). The centrality of metrical structure in signaling information structure: A probabilistic perspective. Language, 86, 1-42.

Chollet, F. (2015). Keras deep learning libraries for Python, version 2.0.8. https://github.com/kerasteam/keras

Church, R., Bernhardt, B., Pichora-Fuller, K., \& Shi, R. (2005). Infant-directed speech: Final syllable lengthening and rate of speech. Journal of The Canadian Acoustical Association, 33, 13-19.

Clark, A. (2013). Whatever next? Predictive brains, situated agents, and the future of cognitive science. Behavioral and Brain Sciences, 36, 181-204.

Cole, J., Mo, Y., \& Hasegawa-Johnson, M. (2010). Signal-based and expectation-based factors in the perception of prosodic prominence. Laboratory Phonology, 1, 425-452.

Cooper, R. P., \& Aslin, R. N. (1990). Preference for infant-directed speech in the first month after birth. Child Development, 61, 1584-1595.

Cristia, A., Dupoux, E., Gurven, M., \& Stieglitz, J. (in press). Child-directed speech is infrequent in a forager-farmer population: A time allocation study. Child Development, online pre-print, http://doi.org/10.1111/cdev.12974.

Cutler, A., \& Foss, D. J. (1977). On the role of sentence stress in sentence processing. Language and Speech, 20, 1-10.

Dale, P. S., \& Fenson, L. (1996). Lexical development norms for young children. Behavioral Research Methods, Instruments, \& Computers, 28, 125-127.

de Boer, B., \& Kuhl, P. (2003). Investigating the role of infant-directed speech with a computer model. Acoustic Research Letters Online, 4, 129-134.

Fenson, L., Marchman, V. A., Thal, D., Dale, P., Reznick, J. S., \& Bates, E. (2007). MacArthur-Bates Communicative Development Inventories: User's Guide and Technical Manual. 2nd Edition. Baltimore, MD: Brookes Publishing Co. 
Fernald, A. (1985). Four-month-old infants prefer to listen to motherese. Infant Behavior and Development, 8, 181-195.

Fernald, A. (1989). Intonation and Communicative Intent in Mothers' Speech to Infants: Is the melody the message? Child Development, 60, 1497-1510.

Fernald, A., Taeschner, T., Dunn, J., Papousek, M., de Boysson-Bardies, B., \& Fukui, I. (1989). A crosslanguage study of prosodic modifications in mothers' and fathers' speech to preverbal infants. Journal of Child Language, 16, 477-501.

Fernald, A., \& Mazzie, C. (1991). Prosody and focus in speech to infants and adults. Developmental Psychology, 27, 209-221.

Friston, K. (2010). The free-energy principle: a unified brain theory? Nature Reviews Neuroscience, 11, $127-138$.

Friston, K., \& Kiebel, S. (2009). Cortical circuits for perceptual inference. Neural Networks, 22, 10931104.

Garnica, O. K. (1977). Some prosodic and paralinguistic features of speech to young children. In C. E. Snow \& C. A. Ferguson (Eds.): Talking to children: Language input and acquisition (pp. 6388). Cambridge, UK: Cambridge University Press.

Ghitza, O. (2011). Linking speech perception and neurophysiology: speech decoding guided by cascaded oscillators locked to the input. Frontiers in Psychology, 2, 1-13.

Giraud, A-L., \& Poeppel, D. (2012). Cortical oscillations and speech processing: emerging computational principles and operations. Nature Neuroscience, 15, 511-517.

Graf Estes, K., \& Hurley, K. (2013). Infant-directed prosody helps infants to map sounds to meanings. Infancy, 18, 10.1111/infa.12006.

Grieser, D., \& Kuhl, P. K. (1988). Maternal speech to infants in a tonal language: support for universal prosodic features in motherese. Developmental Psychology, 24, 14-20.

Gross, J., Hoogenboom. N., Thut, G., Schyns, P., Panzeri, S., Belin, P., \& Garrod, S. (2013). Speech rhythms and multiplexed oscillatory sensory coding in the brain. PLOS Biology, 11(12), e1001752.

Helmholtz, H. (1860/1962). Handbuch der physiologischen optik. Southall, J. P. C. (Ed.), English translation, Vol. 3. New York: Dover.

Hirsh-Pasek, K., Kemler Nelson, D. G., Jusczyk, P. W., Cassidy, K. W., Druss, B., \& Kennedy, L. (1987). Clauses are perceptual units for young infants. Cognition, 26, 269-286.

Itti, L., \& Baldi, P. (2009). Bayesian surprise attracts human attention. Vision Research, 49, 1295-1306. 
Jurafsky, D. (1996). A probabilistic model of lexical and syntactic access and disambiguation. Cognitive Science, 20, 137-194.

Jurafsky, D., Bell, A., Gregory, M., \& Raymond, W. D. (2001). Probabilistic relations between words: Evidence from reduction in lexical production. Typological studies in language, 45, 229-254.

Jusczyk, P. W. (1993). From general to language specific capacities: The WRAPSA model of how speech perception develops. Journal of Phonetics, 21, 3-28.

Kakouros, S., \& Räsänen, O. (2016). Perception of sentence stress in speech correlates with the temporal unpredictability of prosodic features. Cognitive Science, 40, 1739-1774.

Kakouros, S., Salminen, N., \& Räsänen, O. (2018). Making predictable with style - Behavioral and electrophysiological evidence for the critical role of prosodic expectations in the perception of prominence in speech. Neuropsychologia, 109, 181-199.

Kidd, C., Piantadosi, S. T., \& Aslin, R. N. (2012). The Goldilocks effect: human infants allocate attention to visual sequences that are neither too simple nor too complex. PLoS ONE, 7(5), e36399.

Kidd, C., Piantadosi, S., T., \& Aslin, R. N. (2014). The Goldilocks effect in infant auditory attention. Child Development, 85, 1795-1804.

Kirchhoff, K., \& Schimmel, S. (2005). Statistical properties of infant-directed versus adult-directed speech: Insights from speech recognition. Journal of the Acoustical Society of America, 117, $2238-2246$.

Kuhl, P. K., Andruski, J. E., Chistovich, I. A., Chistovich, L. A., Kozhevnikova, E. V., Ryskina, V. L., ... \& Lacerda, F. (1997). Cross-language analysis of phonetic units in language addressed to infants. Science, 277, 684-686.

Kuhl, P. K., \& Miller, J. D. (1982). Discrimination of auditory target dimensions in the presence or absence of variation in a second dimension by infants. Perception \& Psychophysics, 31, 279292.

Li, X., \& Ren, G. (2012). How and when accentuation influences temporally selective attention and subsequent semantic processing during on-line spoken language comprehension: An ERP study. Neuropsychologia, 50, 1882-1894.

Liu, L., \& Kager, R. (2014). Perception of tones by infants learning a non-tone language. Cognition, $133,385-394$.

Ma, W., Golinkoff, R., Houston, D., \& Hirsh-Pasek, K. (2011). Word learning in infant- and adultdirected speech. Language Learning and Development, 7, 185-201. 
Magne, C., Astèsano, C., Lacheret-Dujour, A., Morel, M., Alter, K., \& Besson, M. (2005). On-line processing of "pop-out” words in spoken French dialogues. Journal of Cognitive Neuroscience, $15,740-756$.

Martin, A., Schatz, T., Versteegh, M., Miyazawa, K., Mazuka, R., Dupoux, E., \& Cristia, A. (2015). Mothers speak less clearly to infants than to adults: A comprehensive test of the hyperarticulation hypothesis. Psychological Science, 26, 341-347.

McMurray, B., Kovack-Lesh, K., Goodwin, D., \& McEchron, W. (2013). Infant directed speech and the development of speech perception: Enhancing development or an unintended consequence? Cognition, 129, 362-378.

Mehler, J., Dupoux, E., \& Segui, J. (1990). Constraining models of lexical access: The onset of word recognition. In G. Altmann (Ed.) Cognitive Models of Speech Processing, (pp. 236-262) Cambridge Mass: MIT Press.

Nazzi, T., Bertoncini, J., \& Mehler, J. (1998). Language discrimination by newborns: toward an understanding of the role of rhythm. Journal of Experimental Psychology, 24, 756-766.

Pegg, J. E., Werker, J. F., \& McLeod, P. J. (1992). Preference for infant-directed over adult-directed speech: Evidence from 7-week-old infants. Infant Behavior and Development, 15, 325-345.

Penman, R., Cross, T., Milgrom-Friedman, J., \& Meares, R. (1983). Mothers' speech to prelingual infants: a pragmatic analysis. Journal of Child Language, 10, 17-34.

Phillips, J. R. (1973). Syntax and vocabulary of mothers' speech to young children: Age and sex comparisons. Child Development, 44, 182-185.

Piazza, E. A., Iordan, M. C., \& Lew-Williams, C. (2017). Mothers consistently alter their unique vocal fingerprints when communicating with infants. Current Biology, 27, 1-6.

Ranganath, C., \& Rainer, G. (2003). Neural mechanisms for detecting and remembering novel events. Nature Reviews Neuroscience, 4, 193-202.

Räsänen, O., Doyle, G., \& Frank, M. C. (2018). Pre-linguistic segmentation of speech into syllable-like units. Cognition, 171, 130-150.

Räsänen, O., \& Saarinen, J. P. (2016). Sequence prediction with sparse distributed hyperdimensional coding applied to the analysis of mobile phone use patterns. IEEE Transactions on Neural Networks and Learning Systems, 27, 1878-1889.

Saffran, J. R., Aslin, R. N., \& Newport, E. L. (1996). Statistical learning by 8-month-old infants. Science, 274, 1926-1928. 
Saffran, J. R. (2003). Statistical language learning: Mechanisms and constraints. Current Directions in Psychological Science, 12, 110-114.

Sanford, A. J. S., Sanford, A. J., Molle, J., \& Emmot, C. (2006). Shallow processing and attention capture in written and spoken discourse. Discourse Processes, 42, 109-130.

Saul, L., \& Pereira, F. (1997). Aggregate and mixed-order Markov models for statistical language processing. In Proc. 2nd Conf. Empirical Methods Natural Language Processing, Providence, RI, USA, Aug. 1997, pp. 81-89.

Schuller, B., Batliner, A., Steidl, S., \& Seppi, D. (2011). Recognising realistic emotions and affect in speech: State of the art and lessons learnt from the first challenge. Speech Communication, 53, $1062-1087$.

Shannon, C. (1948). A mathematical theory of communication. The Bell System Technical Journal, 27, $379-423$.

Shattuck-Hufnagel, S., \& Turk, A. E. (1996). A prosody tutorial for investigators of auditory sentence processing. Journal of Psycholinguistic Research, 25, 193-247.

Schneidman, L. \& Goldin-Meadow, S. (2012). Language input and acquisition in a Mayan village: how important is directed speech? Developmental Science, 15, 659-673.

Singh, L., Morgan, J. L., \& Best, C. T. (2002). Infants' listening preferences: Baby talk or happy talk? Infancy, 3, 365-394.

Smith, L., \& Yu, C. (2008). Infants rapidly learn word-referent mappings via cross-situational statistics. Cognition, 106, 1558-1568.

Snow, C. E. (1977). The development of conversation between mothers and babies. Journal of Child Language, 4, 1-22.

Soderstrom, M. (2007). Beyond babytalk: Re-evaluating the nature and content of speech input to preverbal infants. Developmental Review, 27, 501-532.

Soderstrom, M., Conwell, E., Feldman, N., \& Morgan, J. (2009). The learner as statistician: three principles of computational success in language acquisition. Developmental Science, 12, 409411.

Song, J., Demuth, K., \& Morgan, J. (2010). Effects of acoustic properties of infant-directed speech on infant word recognition. Journal of the Acoustical Society of America, 128, 389-400.

Spinelli, M., Fasolo, M., \& Mesman, J. (2017). Does prosody make the difference? A meta-analysis on relations between prosodic aspects of infant-directed speech and infant outcomes. Developmental Review, 44, 1-18. 
Steinhauer, K., Alter, K., \& Friederici, A. D. (1999). Brain potentials indicate immediate use of prosodic cues in natural speech processing. Nature Neuroscience, 2, 191-196.

Stern, D. N., Spieker, S., Barnett, R. K., \& MacKain, K. (1983). The prosody of maternal speech: Infant age and context related changes. Journal of Child Language, 10, 1-15.

Swingley, D. (2005). Statistical clustering and the contents of the infant vocabulary. Cognitive Psychology, 50, 86-132.

[dataset] The ManyBabies Consortium (2017). Quantifying the sources of variability in infancy research using the infant-directed speech preference. Manuscript under review. https://osf.io/re95x/

Thiessen, E. D., Hill, E. A., \& Saffran, J. R. (2005). Infant-directed speech facilitates word segmentation. Infancy, 7, 53-71.

Trainor, L. J., Austin, C. M., \& Desjardins, R. N. (2000). Is infant-directed speech prosody a result of the vocal expression of emotion? Psychological Science, 11, 188-195.

Tsuchida, T., \& Cottrell, G. W. (2012). Auditory saliency using natural statistics. Proceedings of the 34th Annual Conference of the Cognitive Science Society (CogSci-2012), Sapporo, August 1-4 (pp. 1048-1053).

Villing, R., Ward, T., \& Timoney, J. (2006). Performance limits for envelope-based automatic syllable segmentation. Proc. ISSC-2006, Dublin, Ireland, pp. 521-526.

Wang, L., Bastiaansen, M., Yang, Y., \& Hagoort, P. (2011). The influence of information structure on the depth of semantic processing: How focus and pitch accent determine the size of the N400 effect. Neuropsychologia, 49, 813-820.

Watson, D., Arnold, J., \& Tanenhaus, M. (2008). Tic Tac TOE: Effects of predictability and importance on acoustic prominence in language production. Cognition, 106, 1548-1557.

Weisleder, A., \& Fernald, A. (2013). Talking to children matters: Early language experience strengthens processing and builds vocabulary. Psychological Science, 24, 2143-2152.

Zahorian, S., \& Hu H. (2008). A spectral/temporal method for robust fundamental frequency tracking. The Journal of The Acoustical Society of America, 123, 4559-4571.

Zarcone, A., van Schijndel, M., Vogels, J., \& Demberg, V. (2016). Salience and attention in surprisalbased accounts of language processing. Frontiers in Psychology, 7, article no. 844.

Zhang, L., Tong, M. H., Marks, T. K., Shan, H., \& Cottrell, G. W. (2008). SUN: A Bayesian framework for saliency using natural statistics. Journal of Vision, 8, 1-20. 


\section{Appendix A: Full results for the MOCM and LSTM models on syllabic and fixed-frame $\mathrm{FO}$ representations.}
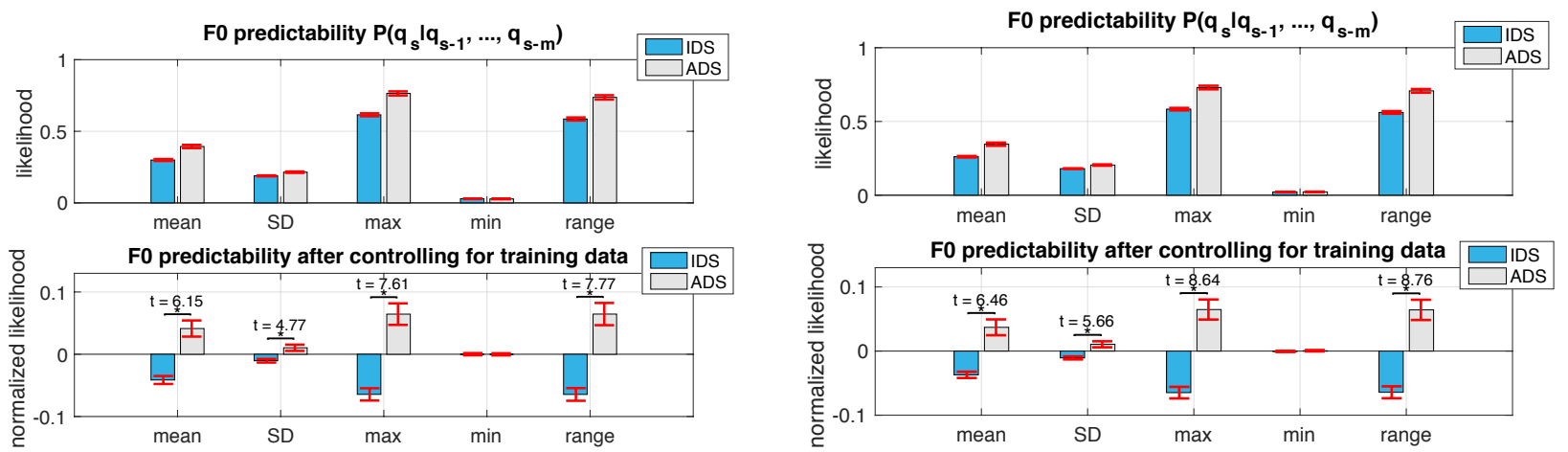

Figure A1: Mixed-order Markov chain model (MOCM) results for syllabic (left) and uniform segments (right).
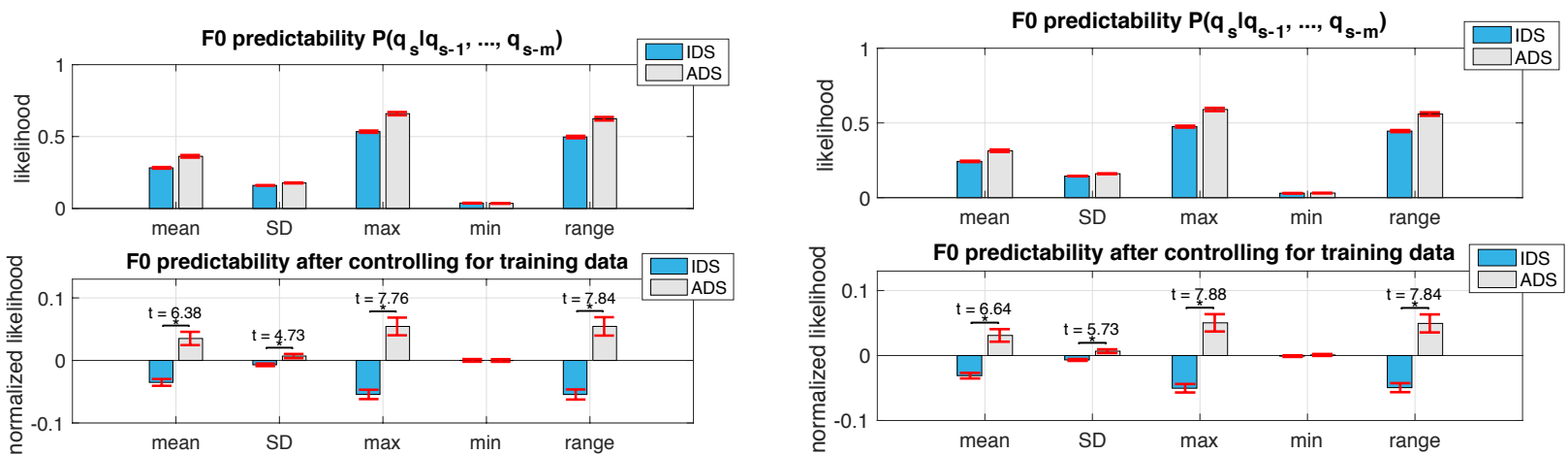

Figure A2: Long short-term memory (LSTM) results for syllabic (left) and uniform segments (right).

Figure A1 shows the results from the comparison between IDS and ADS in terms of F0 predictability and raw F0 values using the MOCM model, while Figure A2 shows the corresponding results for LSTM. In both figures, left panel shows the results from syllabic segments and right panel shows the corresponding results using fixed 100-ms segments for F0 modeling. Top panel: Utterance-level statistical descriptors of F0 predictability, averaged across all ADS/IDS utterances. Bottom panel: F0 predictability after controlling for the amount of matching training data for each utterance. Error bars denote \pm 1 SE across all talkers. Significant differences between IDS and ADS are denoted with asterisks and related t-values (paired t-test, $d f=10$, and using significance level $p<0.05$; Holm-Bonferroni correction for the ten comparisons is used in order to maintain compatibility with the main analyses in Fig. 4 that also include tests for standard F0 descriptors). 